\title{
Comparison between two models of absorption of matter waves by a thin time-dependent barrier
}

\author{
Maximilien Barbier, ${ }^{1}$ Mathieu Beau, ${ }^{2,3}$ and Arseni Goussev ${ }^{1}$ \\ ${ }^{1}$ Department of Mathematics and Information Sciences, Northumbria University, Newcastle Upon Tyne NE1 8ST, United Kingdom \\ ${ }^{2}$ Department of Physics, University of Massachusetts, Boston, Massachusetts 02125, USA \\ ${ }^{3}$ Dublin Institute for Advanced Studies, School of Theoretical Physics, 10 Burlington Road, Dublin 4, Ireland
}

(Received 23 October 2015; published 30 November 2015)

\begin{abstract}
We report a quantitative, analytical, and numerical comparison between two models of the interaction of a nonrelativistic quantum particle with a thin time-dependent absorbing barrier. The first model represents the barrier by a set of time-dependent discontinuous matching conditions, which are closely related to Kottler boundary conditions used in stationary-wave optics as a mathematical basis for Kirchhoff diffraction theory. The second model mimics the absorbing barrier with an off-diagonal $\delta$ potential with a time-dependent amplitude. We show that the two models of absorption agree in their predictions in a semiclassical regime, the regime readily accessible in modern experiments with ultracold atoms.
\end{abstract}

DOI: 10.1103/PhysRevA.92.053630

PACS number(s): 03.75.-b, 37.10.Vz, 03.65.Nk, 42.25.Fx

\section{INTRODUCTION}

The concept of matter-wave absorption often proves valuable in modeling irreversible escape or leakage of a quantum wave function from a practically "interesting" part of the system's Hilbert space into the complementary "uninteresting" part. A representative example is an inelastic atomic (or molecular) collision process, in which a colliding atom may end up ionized (or a molecule dissociated), thus becoming invisible for a detector. An analytical or numerical description of such a process may be significantly simplified by restricting the full wave function to a subspace of detectable states and mimicking its coupling to the complementary subspace of undetectable (ionized or dissociated) states by endowing the system's Hamiltonian with such non-Hermitian features as absorbing complex potentials [1] or absorbing boundary conditions [2-6].

The present study is devoted to a problem of the motion of a one-dimensional quantum particle in the presence of a pointlike absorber whose absorbing properties change in the course of time. On a practical side, this problem models, for instance, a passage of an atom through a partially ionizing laser light sheet of time-dependent intensity. On a more fundamental level, the problem is a generalization of the celebrated Moshinsky shutter problem [7-9], which is a paradigm of the theory of quantum transients [10-13]. As first shown by Moshinsky, a sudden removal of a completely absorbing pointlike barrier may give rise to well-pronounced oscillations of the amplitude of the particle's wave function and the mathematical structure of these oscillations is closely analogous to that of intensity fringes observed in optical diffraction of light at apertures with straight edges. This temporal quantum phenomenon is commonly referred to as diffraction in time. In addition to its purely theoretical interest, diffraction in time has been at the heart of many experimental studies [14-18].

Here we consider a one-dimensional quantum particle characterized by a wave function $\Psi(x, t)$, with $x$ and $t$ denoting the spatial coordinate and time, respectively. As we will be dealing with an absorbing system, the norm of the wave function may generally be smaller than unity, i.e., $\int_{-\infty}^{+\infty} d x|\Psi(x, t)|^{2} \leqslant 1$. We assume, however, that the initial state $\Psi_{0}(x) \equiv \Psi(x, 0)$ is normalized to unity, $\int_{-\infty}^{+\infty} d x\left|\Psi_{0}(x)\right|^{2}=1$, and is spatially localized, for the concreteness, to the left of the origin, so that $\Psi_{0}(x)=0$ for all $x \geqslant 0$. We further imagine that a pointlike barrier is positioned at $x=0$; the barrier is such that it partly absorbs matter waves passing through it and that the proportion of the amount absorbed to the amount transmitted depends on time $t$. (Below we will present a more concrete definition of the absorption process.) The central aim of the present work is to compare two different approaches that allow one to evaluate the particle's wave function $\Psi(x, t)$ in the transmission region $x>0$ at time $t>0$.

The first approach, which we will refer to as the aperture function model (AFM), was originally devised in Refs. [19,20]. It is based on modeling the absorbing barrier by means of discontinuous time-dependent boundary conditions at $x=0$, connecting the values of the wave function and its spatial derivative across the barrier. The main strength of the AFM is that it is exactly solvable [20]. This quality of the model allows for accurate and essentially analytical evaluation of the part of the wave function transmitted through the barrier. In particular, the AFM has been recently used to advance absorption-based displacement, splitting, squeezing, and cooling of atomic wave packets [21]. An apparent weakness of the AFM, however, is the absence of a first-principle derivation of the absorbing boundary conditions. This is why a careful comparison between the AFM and some first-principles model of time-dependent absorption is much needed.

The second approach to the problem, termed here the $\delta$-potential model (DPM), is a time-dependent extension of an atom-laser system studied in Ref. [22]. In this model, the moving particle is a two-level atom, with the internal states labeled $|1\rangle$ and $|2\rangle$. The atom is regarded as detectable (visible) if it is in the internal state $|1\rangle$ and undetectable (invisible or absorbed) if it is in $|2\rangle$. Initially, the atom is detectable and its total state is the product state $\Psi_{0}(x)|1\rangle$. In the course of its motion, the atom interacts with a time-dependent off-diagonal $\delta$ potential, representing an absorbing barrier, whose role is to mix populations of the internal states $|1\rangle$ and $|2\rangle$. As a result of this interaction, the total state of the atom at time $t>0$ will generally be a linear combination of the two internal states. It is the projection on $|1\rangle$ that determines the 
part of the wave function that has not been absorbed by the barrier. An important advantage of the DPM over the AFM is that the former has a solid first-principles justification. Its main disadvantage, however, is that, except for a few special cases, the DPM does not admit analytical treatment and the transmitted wave function has to be computed numerically.

In this paper we make a comparison between the AFM and DPM. We show that in the semiclassical (short-wavelength) regime the two models agree in their predictions of the wave function transmitted through the barrier. In an atom-optics setting, this semiclassical regime is of special importance as it covers a wide range of experimentally relevant parameters. Outside the semiclassical regime, however, we generally find quantitative discrepancies between the predictions of the two models.

The paper is organized as follows. In Sec. II we give a detailed definition of the two models of absorption, the AFM and DPM, and hypothesize about a connection between them. A semiclassical justification of the connection is presented in Sec. III. A numerical study of the connection, both within and outside the semiclassical regime, is reported in Sec. IV. Finally, in Sec. V we discuss our results and make concluding remarks. Some technical details are deferred to the Appendix.

\section{STATEMENT OF THE PROBLEM}

In this section we define, in full detail, two mathematical models describing the motion of a nonrelativistic quantum particle in the presence of a time-dependent absorbing barrier and outline our strategy for making a comparison between them.

\section{A. Aperture function model}

We start by summarizing the AFM, originally developed in Refs. [19,20]. As specified in Sec. I, we consider a quantum particle initially described by a wave function $\Psi_{0}(x)$, which is assumed to be entirely localized on the half-line $x<0$. A time-dependent absorbing barrier is positioned at $x=0$. In a time $t>0, \Psi_{0}(x)$ evolves into a wave function $\Psi_{\mathrm{AFM}}(x, t)$. Below we define the laws governing this evolution.

In the AFM model, the wave function $\Psi_{\mathrm{AFM}}$ is taken to satisfy the time-dependent Schrödinger equation at all times and everywhere away from the barrier, i.e.,

$$
\left(i \frac{\partial}{\partial \tau}+\frac{\hbar}{2 m} \frac{\partial^{2}}{\partial x^{2}}\right) \Psi_{\mathrm{AFM}}(x, \tau)=0
$$

for $0<\tau<t$ and both $x<0$ and $x>0$. The relation between the wave function and its spatial derivative at $x<0$ to those at $x>0$ is given by the conditions

$$
\begin{gathered}
\left.\Psi_{\mathrm{AFM}}(x, \tau)\right|_{x=0^{-}} ^{x=0^{+}}=-\left.[1-\chi(\tau)] \Psi_{\text {free }}(x, \tau)\right|_{x=0}, \\
\left.\frac{\partial \Psi_{\mathrm{AFM}}(x, \tau)}{\partial x}\right|_{x=0^{-}} ^{x=0^{+}}=-\left.[1-\chi(\tau)] \frac{\partial \Psi_{\text {free }}(x, \tau)}{\partial x}\right|_{x=0},
\end{gathered}
$$

satisfied for $0<\tau<t$. Here $\Psi_{\text {free }}(x, \tau)$ is the result of a free propagation of the initial state $\Psi_{0}(x)$ through time $\tau$, i.e.,

$$
\Psi_{\text {free }}(x, \tau)=\int_{-\infty}^{+\infty} d x^{\prime} K_{0}\left(x-x^{\prime}, \tau\right) \Psi_{0}\left(x^{\prime}\right),
$$

where

$$
K_{0}(\xi, \tau)=\sqrt{\frac{m}{2 \pi i \hbar \tau}} \exp \left(i \frac{m \xi^{2}}{2 \hbar \tau}\right)
$$

is the free-particle propagator. The real-valued function $\chi(\tau)$ is the aperture function of the barrier and is defined in the following way. The values of $\chi$ range between 0 and 1 , with 0 representing the situation when the barrier absorbs entirely all incident matter waves (completely absorbing, nontransparent barrier) and 1 corresponding to the unobstructed free-particle motion (perfectly transparent barrier). More generally, $\chi^{2}(\tau)$ is taken to be an instantaneous (pertinent to the barrier at time $\tau$ ) transmission probability defined with respect to the initial state $\Psi_{0}(x)$. The matching conditions, given by Eqs. (2) and (3), mimic the action of a time-dependent absorbing barrier; these conditions are a time-dependent quantum-mechanical generalization of the black-screen boundary conditions originally put forward by Kottler as a way of providing a solid mathematical basis for Kirchhoff diffraction theory [23-25]. Finally, in addition to the Schrödinger equation (1) and the matching conditions (2) and (3), the wave function is required to satisfy Dirichlet boundary conditions at infinity $\lim _{x \rightarrow \pm \infty} \Psi_{\mathrm{AFM}}(x, \tau)=0$ and the initial condition $\Psi_{\mathrm{AFM}}(x, 0)=\Psi_{0}(x)$.

The main benefit of the AFM, formulated above, is that it has a unique exact solution, which in the transmission region, for $x>0$, can be written as [20]

$$
\Psi_{\mathrm{AFM}}(x, t)=\int_{-\infty}^{0} d x^{\prime} K_{\mathrm{AFM}}\left(x, x^{\prime}, t\right) \Psi_{0}\left(x^{\prime}\right),
$$

with

$$
\begin{aligned}
K_{\mathrm{AFM}}\left(x, x^{\prime}, t\right)= & \frac{1}{2} \int_{0}^{t} d \tau \chi(\tau)\left(\frac{x}{t-\tau}-\frac{x^{\prime}}{\tau}\right) \\
& \times K_{0}(x, t-\tau) K_{0}\left(x^{\prime}, \tau\right) .
\end{aligned}
$$

The propagator $K_{\mathrm{AFM}}$ admits the following physical interpretation. The amplitude of a particle's passage from a point $x^{\prime}<0$ to a point $x>0$ in time $t$ is determined by a superposition of a continuous family of paths parametrized by $\tau$. Each of these paths consists of (i) a free flight from $x^{\prime}$ to the barrier in time $\tau$, (ii) a modulation of the amplitude by a factor proportional to the aperture function $\chi(\tau)$ and the mean velocity $\frac{1}{2}\left(\frac{x}{t-\tau}-\frac{x^{\prime}}{\tau}\right)$ at which the particle crosses the barrier, and (iii) another free flight from the barrier to $x$ in the remaining time $t-\tau$.

Finally, we note that the AFM is conceptually similar to other analytical approaches devised to describe the wavefunction transmission through real, reflecting time-dependent barriers (see, e.g., Refs. [26-29]). The relation between quantum propagators for absorbing and reflecting barriers has been recently discussed in Ref. [30].

\section{B. The $\delta$-potential model}

In Ref. [22], an exact propagator was obtained describing the motion of an atom interacting with a pointlike $\delta$-potential laser whose frequency was in resonance with a given interatomic transition. (The corresponding problem for a laser with a semi-infinite spatial extent was studied in Ref. [31].) Here we formulate an extended version of the pointlike atom-laser interaction model in which the intensity of the $\delta$ laser is allowed 
to change in time in accordance with an externally prescribed protocol.

To this end, we consider a two-level atom, with the internal states labeled $|1\rangle$ and $|2\rangle$. At any time $\tau$, the full state of the atom can be written as $\psi_{1}(x, \tau)|1\rangle+\psi_{2}(x, \tau)|2\rangle$, with $\psi_{1}$ and $\psi_{2}$ representing the spatial parts of the state. The atom is initially prepared in the state defined by $\psi_{1}(x, 0)=\Psi_{0}(x)$ and $\psi_{2}(x, 0)=0$. In the course of its time evolution, the atom propagates freely everywhere in space except for the point $x=0$, at which a barrier is placed. The barrier is represented by an off-diagonal $\delta$ potential with a time-dependent amplitude $V(x, \tau)=\hbar \Omega(\tau) \delta(x)(|1\rangle\langle 2|+| 2\rangle\langle 1|)$. The amplitude $\Omega(\tau)$ is a positive-valued function of time quantifying the strength of the atom-laser coupling and directly proportional to the square root of the laser intensity. So, the time evolution of the full atomic state is governed by the time-dependent Schrödinger equation

$$
i \hbar \frac{\partial}{\partial \tau}\left(\begin{array}{l}
\psi_{1} \\
\psi_{2}
\end{array}\right)=H_{\mathrm{DPM}}\left(\begin{array}{l}
\psi_{1} \\
\psi_{2}
\end{array}\right)
$$

for $0<\tau<t$, where

$$
H_{\mathrm{DPM}}=H_{0}+V(x, \tau)
$$

with

$$
H_{0}=-\frac{\hbar^{2}}{2 m} \frac{\partial^{2}}{\partial x^{2}}\left(\begin{array}{ll}
1 & 0 \\
0 & 1
\end{array}\right)
$$

and

$$
V(x, \tau)=\hbar \Omega(\tau) \delta(x)\left(\begin{array}{ll}
0 & 1 \\
1 & 0
\end{array}\right) .
$$

In addition, the full wave function is subject to the usual Dirichlet boundary conditions at infinity $\lim _{x \rightarrow \pm \infty} \psi_{1}(x, \tau)=$ 0 and $\lim _{x \rightarrow \pm \infty} \psi_{2}(x, \tau)=0$.

In the context of the DPM, the internal state $|1\rangle$ represents detectable particles and $|2\rangle$ labels particles absorbed by the barrier. Thus, the atom that at time $t$ has traversed the barrier and remained in its original internal state $|1\rangle$ or, in other words, the particle that has survived the absorbing barrier is described by the wave function

$$
\Psi_{\mathrm{DPM}}(x, t)=\psi_{1}(x, t) .
$$

The wave function $\Psi_{\mathrm{DPM}}$ admits analytical evaluation only in very few special cases. One such case is that of a time-independent potential $\Omega(\tau)=\Omega_{0}$ [22]. Another exactly solvable case corresponds to $\Omega(\tau)=\Omega_{1} / \tau$, with $\Omega_{1}$ being a constant; here the exact propagator is obtained by first making the substitution $\psi_{ \pm}=\psi_{1} \pm \psi_{2}$ to decouple Eq. (8) and then using a known solution for the problem of a one-dimensional particle in the $\tau^{-1} \delta(x)$ potential [32]. However, in general, i.e., for an arbitrary function $\Omega(\tau)$, Eq. (8) can only be tackled by means of a direct numerical integration.

\section{Connection between the two models}

In this paper we make a quantitative comparison between $\Psi_{\mathrm{AFM}}(x, t)$ and $\Psi_{\mathrm{DPM}}(x, t)$ in the transmission region $x>0$. The initial wave function of the particle is taken to be a
Gaussian wave packet

$$
\Psi_{0}(x)=\left(\frac{1}{\pi \sigma^{2}}\right)^{1 / 4} \exp \left[-\frac{\left(x-x_{0}\right)^{2}}{2 \sigma^{2}}+i \frac{m v_{0}}{\hbar}\left(x-x_{0}\right)\right]
$$

where $m$ is the mass of the particle, $x_{0}$ and $v_{0}$ are its average position and velocity, respectively, and $\sigma$ is the spatial extent of the wave packet. Hereinafter we consider $x_{0}<0$, $\left|x_{0}\right| \gg \sigma>0$, and $v_{0}>0$. The reason for our choice of the initial wave function is twofold. On the one hand, having $\Psi_{0}(x)$ given by a simple Gaussian wave packet facilitates analytical treatment of the problem. On the other hand, localized wave packets with a nonzero average velocity can be routinely generated in laboratory experiments with ultracold atoms (see, e.g., Refs. [33-35]).

In order to compare the two models of absorption, the AFM and DPM, we first need to specify a relation $\chi(\tau)=$ $\mathcal{T}(\Omega(\tau)$ ) connecting the aperture function $\chi$ with the atomlaser coupling strength $\Omega$ at any time $0<\tau<t$. Here we do this in the following intuitive way: We take the function $\mathcal{T}\left(\Omega_{0}\right)$ to be the $|1\rangle$-channel transmission amplitude associated with a plane wave of momentum $m v_{0}$ incident upon a $\delta$ barrier of constant strength $\Omega_{0}$. More concretely, we take $\Omega(\tau)=\Omega_{0}$ and consider a scattering state solution to Eq. (8) of the form

$$
\left(\begin{array}{l}
\psi_{1} \\
\psi_{2}
\end{array}\right)=e^{i(k x-\omega t)} \begin{cases}\left(\begin{array}{l}
1 \\
0
\end{array}\right)+\left(\begin{array}{l}
\mathcal{R}_{11} \\
\mathcal{R}_{21}
\end{array}\right) e^{-2 i k x} & \text { for } x<0 \\
\left(\begin{array}{l}
\mathcal{T}_{11} \\
\mathcal{T}_{21}
\end{array}\right) & \text { for } x>0,\end{cases}
$$

with $k=m v_{0} / \hbar$ and $\omega=m v_{0}^{2} / 2 \hbar$. The transmission amplitudes $\mathcal{T}_{11}$ and $\mathcal{T}_{21}$ and the reflection amplitudes $\mathcal{R}_{11}$ and $\mathcal{R}_{21}$ are found in a standard way by requiring the wave function to be continuous and to have a discontinuous spatial derivative at $x=0$. In particular, one can straightforwardly show that $\mathcal{T}_{11}=1 /\left[1+\left(m \Omega_{0} / \hbar k\right)^{2}\right]=1 /\left[1+\left(\Omega_{0} / v_{0}\right)^{2}\right]$. We then take the transmission amplitude $\mathcal{T}_{11}$ as a definition of the function $\mathcal{T}\left(\Omega_{0}\right)$, so that, for a time-dependent $\delta$ barrier, we have

$$
\chi(\tau)=\frac{1}{1+\left[\Omega(\tau) / v_{0}\right]^{2}}
$$

for $0<\tau<t$

Equipped with Eq. (15), it is now feasible to compare the wave functions $\Psi_{\mathrm{AFM}}(x, t)$ and $\Psi_{\mathrm{DPM}}(x, t)$ evolved from the same initial state $\Psi_{0}(x)$, given by Eq. (13). In Sec. III we make this comparison analytically in a semiclassical regime and in the subsequent section, Sec. IV, we go beyond the semiclassical regime by numerically solving Eq. (8) (or by using exact analytical results when available) in various experimentally realistic scenarios. We summarize our results and make concluding remarks in Sec. V.

\section{SEMICLASSICAL REGIME}

The aim of this section is to analytically investigate the validity of Eq. (15) as a proposed connection between the AFM and DPM. Our analytical calculations are performed in a semiclassical regime defined with respect to the initial wave packet $\Psi_{0}(x)$, given by Eq. (13) and characterized by the mean 
position $x_{0}<0$, mean velocity $v_{0}>0$, and spatial dispersion $\sigma$. The semiclassical regime is detailed by the conditions

$$
\sigma \ll\left|x_{0}\right| \lesssim v_{0}\left(t-t_{c}\right) \ll \frac{m \sigma^{2} v_{0}}{\hbar},
$$

where

$$
t_{c} \equiv \frac{\left|x_{0}\right|}{v_{0}}
$$

denotes the time needed for the corresponding classical particle to reach the barrier. The first condition in Eq. (16), $\sigma \ll\left|x_{0}\right|$, specifies that the initial wave packet is well localized around $x_{0}$ and allows us to effectively restrict the support of $\Psi_{0}(x)$ to the half-line $x<0$. The other two conditions $\left|x_{0}\right| \lesssim v_{0}\left(t-t_{c}\right)$ and $v_{0}\left(t-t_{c}\right) \ll m \sigma^{2} v_{0} / \hbar$ ensure that at time $t$ and in the absence of a barrier the wave packet would be localized well inside the transmission region $x>0$. Indeed, the two conditions imply that $\hbar t / m \sigma^{2} \ll 1$, which means that the spatial spreading of the wave packet dictated by the uncertainty principle is negligible during the time $t$; this regime is closely related to the so-called frozen Gaussian approximation [36].

We now want to compare the predictions of the AFM and DPM in the semiclassical regime defined by Eq. (16). The wave function $\Psi_{\mathrm{AFM}}(x, t)$ is related to the initial state $\Psi_{0}(x)$ through Eqs. (6) and (7). Similarly, in the DPM, the state $\psi_{1}(x, t)|1\rangle+\psi_{2}(x, t)|2\rangle$ of the two-level atom is related to its initial state $\Psi_{0}(x)|1\rangle$ through

$$
\left(\begin{array}{l}
\psi_{1}(x, t) \\
\psi_{2}(x, t)
\end{array}\right)=\int_{-\infty}^{0} d x^{\prime} \widehat{K}\left(x, x^{\prime}, t\right)\left(\begin{array}{c}
\Psi_{0}\left(x^{\prime}\right) \\
0
\end{array}\right) .
$$

Here $\widehat{K}$ is a matrix propagator

$$
\widehat{K}\left(x, x^{\prime}, t\right)=\left(\begin{array}{ll}
K_{11}\left(x, x^{\prime}, t\right) & K_{12}\left(x, x^{\prime}, t\right) \\
K_{21}\left(x, x^{\prime}, t\right) & K_{22}\left(x, x^{\prime}, t\right)
\end{array}\right),
$$

whose first component determines the wave function $\Psi_{\mathrm{DPM}}(x, t)$ :

$$
\Psi_{\mathrm{DPM}}(x, t)=\int_{-\infty}^{0} d x^{\prime} K_{11}\left(x, x^{\prime}, t\right) \Psi_{0}\left(x^{\prime}\right) .
$$

In this section we analytically compare the wave functions $\Psi_{\mathrm{AFM}}(x, t)$ and $\Psi_{\mathrm{DPM}}(x, t)$ in two different scenarios. First, in Sec. III A, we address the case of a slowly varying timedependent barrier and within the semiclassical approximation find an explicit expression for the propagator $K_{11}\left(x, x^{\prime}, t\right)$. Using this expression, we show that if $\chi(\tau)$ and $\Omega(\tau)$ are related to each other through Eq. (15), the agreement between the two wave functions $\Psi_{\mathrm{AFM}}(x, t)$ and $\Psi_{\mathrm{DPM}}(x, t)$ is strong in a spatial region around the center of the corresponding free-particle wave packet, i.e., around the point $x=x_{t}$, where the function $x_{\tau}$ is defined as

$$
x_{\tau} \equiv x_{0}+v_{0} \tau \text {. }
$$

Then, in Sec. III B, we analyze the case of a rapidly (in fact, instantaneously) varying barrier and again demonstrate a strong agreement between the two wave functions in a neighborhood of the point $x_{t}$. (We quantify the spatial extent of the neighborhood in Sec. IV.)

\section{A. Slowly varying barrier}

Let us first recall that the full propagator $\widehat{K}\left(x, x^{\prime}, \tau\right)$ must obey the time-dependent Schrödinger equation (8). Therefore, the element $K_{11}\left(x, x^{\prime}, \tau\right)$ satisfies the free-particle time-dependent Schrödinger equation on both sides of the barrier, i.e.,

$$
\left(\frac{\partial^{2}}{\partial x^{2}}+\frac{i}{\alpha} \frac{\partial}{\partial \tau}\right) K_{11}\left(x, x^{\prime}, \tau\right)=0 \quad \text { for } x, x^{\prime} \neq 0,
$$

where $\alpha=\hbar / 2 m$. By definition of a quantum propagator, $K_{11}\left(x, x^{\prime}, \tau\right)$ is subject to the initial condition

$$
K_{11}\left(x, x^{\prime}, 0^{+}\right)=\delta\left(x-x^{\prime}\right) .
$$

Also, Dirichlet boundary conditions imposed on the wave function at infinity require

$$
K_{11}\left(x \rightarrow \pm \infty, x^{\prime}, \tau\right)=0 \text { for } \alpha=-i|\alpha| .
$$

In addition to Eqs. (23) and (24), we also know one matching condition at $x=0$. Indeed, the spatial continuity of the wave function implies that $K_{11}\left(x, x^{\prime}, \tau\right)$ is continuous at $x=0$, so that

$$
\left.K_{11}\left(x, x^{\prime}, \tau\right)\right|_{x=0^{-}} ^{x=0^{+}}=0 .
$$

Therefore, we only lack one additional matching condition at $x=0$ in order to have a well-posed mathematical problem, uniquely determining $K_{11}\left(x, x^{\prime}, \tau\right)$.

Our strategy is as follows. First, within the semiclassical regime and under the assumption of a slowly varying barrier, we find the missing matching condition satisfied by the spatial derivative of $K_{11}$ at $x=0$. Second, we solve the resulting mathematical problem for the propagator, obtaining an explicit expression for $K_{11}\left(x, x^{\prime}, \tau\right)$. Third, using the expression for the propagator, we make a direct comparison between the wave functions $\Psi_{\mathrm{AFM}}(x, t)$ and $\Psi_{\mathrm{DPM}}(x, t)$, finding the two in good agreement.

\section{Matching condition for $\partial_{x} K_{11}\left(x, x^{\prime}, \tau\right)$ at $x=0$}

The full propagator $\widehat{K}\left(x, x^{\prime}, \tau\right)$ satisfies the time-dependent Schrödinger equation $i \hbar \frac{\partial}{\partial \tau} \widehat{K}=H_{\text {DPM }} \widehat{K}$ with $H_{\text {DPM }}=H_{0}+$ $V(x, \tau)$ [cf. Eqs. (8)-(11)]. Denoting the matrix free-particle propagator, corresponding to $H_{0}$, by

$$
\widehat{K}_{0}\left(x-x^{\prime}, \tau\right) \equiv K_{0}\left(x-x^{\prime}, \tau\right)\left(\begin{array}{ll}
1 & 0 \\
0 & 1
\end{array}\right),
$$

we use the time-dependent Lippmann-Schwinger equation [37] to represent $\widehat{K}\left(x, x^{\prime}, \tau\right)$ as a Dyson series:

$$
\begin{aligned}
\widehat{K}\left(x, x^{\prime}, \tau\right)= & \widehat{K}_{0}\left(x-x^{\prime}, \tau\right)-\frac{i}{\hbar} \int_{0}^{\tau} d \tau_{1} \int_{-\infty}^{+\infty} d x^{\prime \prime} \\
& \times \widehat{K}_{0}\left(x-x^{\prime \prime}, \tau-\tau_{1}\right) V\left(x^{\prime \prime}, \tau_{1}\right) \widehat{K}\left(x^{\prime \prime}, x^{\prime}, \tau_{1}\right) \\
& =\widehat{K}_{0}\left(x-x^{\prime}, \tau\right)-i \int_{0}^{\tau} d \tau_{1} \widehat{K}_{0}\left(x, \tau-\tau_{1}\right) \Omega\left(\tau_{1}\right) \\
& \times\left(\begin{array}{ll}
0 & 1 \\
1 & 0
\end{array}\right) \widehat{K}\left(0, x^{\prime}, \tau_{1}\right) \\
& =\widehat{K}_{0}\left(x-x^{\prime}, \tau\right)+\sum_{n=1}^{+\infty} \widehat{K}^{(n)}\left(x, x^{\prime}, \tau\right),
\end{aligned}
$$


where

$$
\begin{aligned}
\widehat{K}^{(n)}\left(x, x^{\prime}, \tau\right)= & (-i)^{n} \int_{0}^{\tau} d \tau_{n} \int_{0}^{\tau_{n}} d \tau_{n-1} \cdots \int_{0}^{\tau_{2}} d \tau_{1} K_{0}\left(x, \tau-\tau_{n}\right) \Omega\left(\tau_{n}\right) K_{0}\left(0, \tau_{n}-\tau_{n-1}\right) \cdots \Omega\left(\tau_{2}\right) \\
& \times K_{0}\left(0, \tau_{2}-\tau_{1}\right) \Omega\left(\tau_{1}\right) K_{0}\left(x^{\prime}, \tau_{1}\right)\left(\begin{array}{ll}
0 & 1 \\
1 & 0
\end{array}\right)^{n} .
\end{aligned}
$$

We can readily see from Eq. (28) that the element $K_{11}^{(n)}\left(x, x^{\prime}, \tau\right) \equiv\left[\widehat{K}^{(n)}\left(x, x^{\prime}, \tau\right)\right]_{11}$ vanishes for odd $n$,

$$
K_{11}^{(n)}\left(x, x^{\prime}, \tau\right)=0 \quad \text { for } n=2 k+1, k \geqslant 0
$$

and for even $n$ is given by

$$
\begin{aligned}
K_{11}^{(n)}\left(x, x^{\prime}, \tau\right)= & (-i)^{n} \int_{0}^{\tau} d \tau_{n} \int_{0}^{\tau_{n}} d \tau_{n-1} \cdots \int_{0}^{\tau_{2}} d \tau_{1} K_{0}\left(x, \tau-\tau_{n}\right) \Omega\left(\tau_{n}\right) K_{0}\left(0, \tau_{n}-\tau_{n-1}\right) \cdots \Omega\left(\tau_{2}\right) K_{0}\left(0, \tau_{2}-\tau_{1}\right) \\
& \times \Omega\left(\tau_{1}\right) K_{0}\left(x^{\prime}, \tau_{1}\right) \quad \text { for } n=2 k, k \geqslant 1 .
\end{aligned}
$$

Therefore, in the rest of this section, we treat $n$ as an even integer, i.e., $n=2 k$ with $k \geqslant 1$.

Let us now calculate the partial derivative of $K_{11}^{(n)}\left(x, x^{\prime}, \tau\right)$ with respect to $x$. To this end, we first rewrite Eq. (30) as

$$
K_{11}^{(n)}\left(\xi_{n+1}, \xi_{0}, \tau_{n+1}\right)=(-i)^{n} \lim _{\xi_{n} \rightarrow 0} \cdots \lim _{\xi_{1} \rightarrow 0} \int_{0}^{\tau_{n+1}} d \tau_{n} \cdots \int_{0}^{\tau_{2}} d \tau_{1}\left(\prod_{j=1}^{n} \Omega\left(\tau_{j}\right)\right)\left(\prod_{j=0}^{n} K_{0}\left(\xi_{j+1}-\xi_{j}, \tau_{j+1}-\tau_{j}\right)\right)
$$

where, in order to simplify the subsequent calculations, we have introduced $\xi_{n+1} \equiv x, \xi_{0} \equiv x^{\prime}, \tau_{n+1} \equiv \tau$, and $\tau_{0} \equiv 0$. Then, differentiating Eq. (31) with respect to $x=\xi_{n+1}$ and introducing the dimensionless parameters $\epsilon_{j} \equiv \xi_{j} /\left|x_{0}\right|$ and $\eta_{j} \equiv \tau_{j} / \tau \equiv$ $\tau_{j} / \tau_{n+1}$, we get, in view of Eq. (5),

$$
\frac{\partial}{\partial x} K_{11}^{(n)}\left(x, x^{\prime}, \tau\right)=\frac{1}{\left|x_{0}\right|} \frac{\partial}{\partial \epsilon_{n+1}} K_{11}^{(n)}\left(\left|x_{0}\right| \epsilon_{n+1},\left|x_{0}\right| \epsilon_{0}, \tau_{n+1} \eta_{n+1}\right)=\lim _{\epsilon_{n} \rightarrow 0} \ldots \lim _{\epsilon_{1} \rightarrow 0} I^{(n)}\left(\epsilon_{0}, \ldots, \epsilon_{n+1}, \eta_{n+1}\right),
$$

with

$$
I^{(n)}\left(\epsilon_{0}, \ldots, \epsilon_{n+1}, \eta_{n+1}\right)=\int_{0}^{\eta_{n+1}} d \eta_{n} \cdots \int_{0}^{\eta_{2}} d \eta_{1} F\left(\eta_{1}, \ldots, \eta_{n}\right) e^{i \lambda \phi\left(\eta_{1}, \ldots, \eta_{n}\right)},
$$

where $\lambda=m x_{0}^{2} / 2 \hbar \tau$, the amplitude $F$ is defined as

$$
F\left(\eta_{1}, \ldots, \eta_{n}\right)=(-i)^{n}\left(\frac{m}{2 i \pi \hbar}\right)^{\frac{n+1}{2}}\left(\prod_{j=1}^{n} \Omega\left(\tau \eta_{j}\right)\right) \frac{i m\left|x_{0}\right|\left(\epsilon_{n+1}-\epsilon_{n}\right)}{\hbar\left(\eta_{n+1}-\eta_{n}\right)} \frac{\tau^{\frac{n-3}{2}}}{\sqrt{\prod_{j=0}^{n}\left(\eta_{j+1}-\eta_{j}\right)}},
$$

and the phase $\phi$ is defined as

$$
\phi\left(\eta_{1}, \ldots, \eta_{n}\right)=\sum_{j=0}^{n} \frac{\left(\epsilon_{j+1}-\epsilon_{j}\right)^{2}}{\eta_{j+1}-\eta_{j}} .
$$

We now compute $I^{(n)}\left(\epsilon_{0}, \ldots, \epsilon_{n+1}, \eta_{n+1}\right)$ in the semiclassical regime, defined by Eq. (16). Keeping in mind that $\lambda \gg 1$ in the semiclassical regime, we evaluate the $n$-dimensional integral in Eq. (33) by using the stationary phase approximation [37].

First, we find the stationary point $\boldsymbol{\eta}^{(s)}=\left(\eta_{1}^{(s)}, \ldots, \eta_{n}^{(s)}\right)$, defined by the system of equations

$$
\left.\frac{\partial \phi}{\partial \eta_{j}}\right|_{\left(\eta_{1}, \ldots, \eta_{n}\right)=\eta^{(s)}}=0
$$

for all $1 \leqslant j \leqslant n$ and the constraint

$$
0<\eta_{1}^{(s)}<\cdots<\eta_{n}^{(s)}<1
$$

The condition given by Eq. (37) is needed to ensure the existence of a neighborhood of the stationary point $\boldsymbol{\eta}^{(s)}$ that is entirely contained inside the integration domain of Eq. (33). It can be straightforwardly verified that the unique solution to Eqs. (36) and (37) is

$$
\eta_{j}^{(s)}=\frac{\sum_{k=1}^{j}\left|\epsilon_{k}-\epsilon_{k-1}\right|}{\sum_{k=1}^{n+1}\left|\epsilon_{k}-\epsilon_{k-1}\right|}
$$

for all $1 \leqslant j \leqslant n$.

The stationary phase evaluation of the integral in Eq. (33) proceeds in the standard way. We restrict the integration domain to a neighborhood of the stationary point $\boldsymbol{\eta}^{(s)}$ and replace the phase $\phi\left(\eta_{1}, \ldots, \eta_{n}\right)$ by its second-order Taylor expansion in powers of $\left(\eta_{j}-\eta_{j}^{(s)}\right)$. Then, assuming that $\Omega(\tau)$ is a slowly, essentially algebraically varying function of time, we replace the function $F\left(\eta_{1}, \ldots, \eta_{n}\right)$ by its value at the stationary point $F\left(\eta_{1}^{(s)}, \ldots, \eta_{n}^{(s)}\right)$ and take it outside the $n$-dimensional integral in Eq. (33). Finally, extending the integration region to $\mathbb{R}^{n}$ and performing the $n$-dimensional Gaussian integration 
[38], we obtain

$$
I^{(n)}\left(\epsilon_{0}, \ldots, \epsilon_{n+1}, \eta_{n+1}\right)=\left(\frac{2 \pi}{\lambda}\right)^{\frac{n}{2}} \frac{F^{(s)} e^{i \lambda \phi^{(s)}+i n \pi / 4}}{\sqrt{\operatorname{det}(\mathcal{H})}} .
$$

Here $F^{(s)} \equiv F\left(\eta_{1}^{(s)}, \ldots, \eta_{n}^{(s)}\right)$ and $\phi^{(s)} \equiv \phi\left(\eta_{1}^{(s)}, \ldots, \eta_{n}^{(s)}\right)$ are the values of the amplitude and phase at the stationary point, respectively, and $\mathcal{H}$ is the $n \times n$ Hessian matrix, with elements defined as

$$
\mathcal{H}_{j k}=\left.\frac{\partial^{2} \phi}{\partial \eta_{j} \partial \eta_{k}}\right|_{\left(\eta_{1}, \ldots, \eta_{n}\right)=\eta^{(s)}},
$$

where $1 \leqslant j \leqslant n$ and $1 \leqslant k \leqslant n$.

Substituting Eq. (35) into Eq. (40), we see that the Hessian matrix is symmetric and tridiagonal and its elements are given by

$$
\mathcal{H}_{j j}=2\left(\sum_{k=1}^{n+1}\left|\epsilon_{k}-\epsilon_{k-1}\right|\right)^{3}\left(\frac{1}{\left|\epsilon_{j}-\epsilon_{j-1}\right|}+\frac{1}{\left|\epsilon_{j+1}-\epsilon_{j}\right|}\right)
$$

for all $1 \leqslant j \leqslant n$,

$$
\mathcal{H}_{j, j+1}=\mathcal{H}_{j+1, j}=-2 \frac{\left(\sum_{k=1}^{n+1}\left|\epsilon_{k}-\epsilon_{k-1}\right|\right)^{3}}{\left|\epsilon_{j+1}-\epsilon_{j}\right|}
$$

for all $1 \leqslant j \leqslant n-1$, and $H_{j k}=0$ for all $|j-k| \geqslant 2$. The determinant of the Hessian matrix is given by (see the Appendix for details of the calculation)

$$
\operatorname{det}(\mathcal{H})=2^{n} \frac{\left(\sum_{k=1}^{n+1}\left|\epsilon_{k}-\epsilon_{k-1}\right|\right)^{3 n+1}}{\prod_{k=1}^{n+1}\left|\epsilon_{k}-\epsilon_{k-1}\right|}
$$

We then use Eqs. (34), (35), (38), and (42) in Eq. (39), substitute the resulting expression for $I^{(n)}\left(\epsilon_{0}, \ldots, \epsilon_{n+1}, \eta_{n+1}\right)$ into Eq. (32), and take the limits $\epsilon_{1} \rightarrow 0, \ldots, \epsilon_{n} \rightarrow 0$ to obtain

$$
\begin{aligned}
\frac{\partial}{\partial x} K_{11}^{(2 k)}\left(x, x^{\prime}, \tau\right)= & \operatorname{sgn}(x) \frac{m\left(|x|-x^{\prime}\right)}{i \hbar \tau}(-1)^{k+1} K_{0}\left(|x|-x^{\prime}, \tau\right) \\
& \times\left[\frac{\tau^{2}}{\left(|x|-x^{\prime}\right)^{2}} \Omega\left(\frac{x^{\prime}}{x^{\prime}-|x|} \tau\right)^{2}\right]^{k}
\end{aligned}
$$

for all $k \geqslant 1$. Here $\operatorname{sgn}(x)=x /|x|$ denotes the sign function.

Differentiating Eq. (27) with respect to $x$ and combining the resulting expression with Eqs. (29) and (43), we find

$$
\begin{aligned}
\frac{\partial}{\partial x} K_{11}\left(x, x^{\prime}, \tau\right)= & \frac{\partial K_{0}}{\partial x}+\sum_{k=1}^{+\infty} \frac{\partial}{\partial x} K_{11}^{(2 k)}\left(x, x^{\prime}, \tau\right) \\
= & \frac{\partial K_{0}}{\partial x}+\operatorname{sgn}(x) \frac{m\left(|x|-x^{\prime}\right)}{i \hbar \tau} K_{0}\left(|x|-x^{\prime}, \tau\right) \\
& \times \sum_{k=1}^{+\infty}(-1)^{k+1}\left[\frac{\tau^{2}}{\left(|x|-x^{\prime}\right)^{2}} \Omega\left(\frac{x^{\prime}}{x^{\prime}-|x|} \tau\right)^{2}\right]^{k} .
\end{aligned}
$$

Then Eq. (44) readily gives us the jump of the spatial derivative at the origin

$$
\begin{aligned}
& \left.\frac{\partial}{\partial x} K_{11}\left(x, x^{\prime}, \tau\right)\right|_{x=0^{-}} ^{x=0^{+}} \\
& \quad=-2 \frac{m x^{\prime}}{i \hbar \tau} K_{0}\left(x^{\prime}, \tau\right) \sum_{k=1}^{+\infty}(-1)^{k+1}\left[\frac{\tau^{2}}{x^{\prime 2}} \Omega(\tau)^{2}\right]^{k} .
\end{aligned}
$$

Finally, using the identities $K_{0}\left(x^{\prime}, \tau\right) m x^{\prime} / i \hbar \tau=\partial_{x} K_{0}(x-$ $\left.x^{\prime}, \tau\right)\left.\right|_{x=0}$ and $\sum_{k=1}^{+\infty}(-1)^{k+1} z^{k}=z /(1+z)$ for any $z>0$, we rewrite Eq. (45) as

$$
\left.\frac{\partial}{\partial x} K_{11}\left(x, x^{\prime}, \tau\right)\right|_{x=0^{-}} ^{x=0^{+}}=-\left.\kappa\left(x^{\prime}, \tau\right) \frac{\partial}{\partial x} K_{0}\left(x-x^{\prime}, \tau\right)\right|_{x=0}
$$

with

$$
\kappa\left(x^{\prime}, \tau\right)=\frac{2}{1+\left(\frac{x^{\prime}}{\tau \Omega(\tau)}\right)^{2}} .
$$

Equation (46) constitutes the desired matching condition for the spatial derivative of the propagator.

\section{Derivation of $K_{11}\left(x, x^{\prime}, \tau\right)$}

We now solve the initial-boundary-value problem formed by the time-dependent Schrödinger equation (22), the initial condition (23), and the boundary conditions (24), (25), and (46). Our method of choice is the method of Laplace transforms. In the rest of this section we adopt the following notation for the Laplace transform of a function $f(\tau)$ :

$$
\bar{f}(s)=\mathscr{L}[f(\tau)]=\int_{0}^{+\infty} d \tau e^{-s \tau} f(\tau) .
$$

Taking the Laplace transform of both sides of Eq. (22), we obtain

$$
\frac{\partial^{2}}{\partial x^{2}} \bar{K}_{11}\left(x, x^{\prime}, s\right)+\frac{i s}{\alpha} \bar{K}_{11}\left(x, x^{\prime}, s\right)=\frac{i}{\alpha} \delta\left(x-x^{\prime}\right)
$$

for $x, x^{\prime} \neq 0$. The structure of Eq. (49) implies that $\bar{K}_{11}\left(x, x^{\prime}, s\right)$ is continuous and $\frac{\partial}{\partial x} \bar{K}_{11}\left(x, x^{\prime}, s\right)$ is discontinuous at $x=x^{\prime}$. Therefore, Eq. (49) is equivalent to the homogeneous equation

$$
\frac{\partial^{2}}{\partial x^{2}} \bar{K}_{11}\left(x, x^{\prime}, s\right)+\frac{i s}{\alpha} \bar{K}_{11}\left(x, x^{\prime}, s\right)=0
$$

for $x, x^{\prime} \neq 0$ and $x \neq x^{\prime}$, with the matching conditions

$$
\left.\bar{K}_{11}\left(x, x^{\prime}, s\right)\right|_{\substack{x=x^{\prime+} \\ x=x^{\prime-}}} ^{x}=0
$$

and

$$
\left.\frac{\partial}{\partial x} \bar{K}_{11}\left(x, x^{\prime}, s\right)\right|_{x=x^{\prime-}} ^{x=x^{\prime+}}=\frac{i}{\alpha} .
$$

Also, taking the Laplace transform of Eqs. (24), (25), and (46), we obtain, respectively,

$$
\begin{gathered}
\bar{K}_{11}\left(x \rightarrow \pm \infty, x^{\prime}, s\right)=0 \quad \text { for } \alpha=-i|\alpha|, \\
\left.\bar{K}_{11}\left(x, x^{\prime}, s\right)\right|_{x=0^{-}} ^{x=0^{+}}=0,
\end{gathered}
$$


and

$$
\left.\frac{\partial}{\partial x} \bar{K}_{11}\left(x, x^{\prime}, s\right)\right|_{x=0^{-}} ^{x=0^{+}}=\bar{Q}(s),
$$

where

$$
\begin{aligned}
\bar{Q}(s) & =\mathscr{L}\left[-\left.\kappa\left(x^{\prime}, \tau\right) \frac{\partial}{\partial x} K_{0}\left(x-x^{\prime}, \tau\right)\right|_{x=0}\right] \\
& =\frac{i x^{\prime}}{2 \alpha} \int_{0}^{+\infty} d \tau e^{-s \tau} \kappa\left(x^{\prime}, \tau\right) \frac{K_{0}\left(x^{\prime}, \tau\right)}{\tau} .
\end{aligned}
$$

Equations (50) and (51) uniquely specify the function $\bar{K}_{11}\left(x, x^{\prime}, s\right)$.

Recalling that we are only interested in the case of $x^{\prime}<0$, we define the following three spatial intervals (with respect to a fixed value of $x^{\prime}$ ): the first interval $\mathcal{R}_{1}$ is the set of all points $x$ such that $-\infty<x<x^{\prime}$, the second interval $\mathcal{R}_{2}$ corresponds to $x^{\prime}<x<0$, and the third interval $\mathcal{R}_{3}$ corresponds to $0<$ $x<+\infty$. The general solution of Eq. (50) is given by

$$
\bar{K}_{11}\left(x, x^{\prime}, s\right)=A_{j} e^{k_{+} x}+B_{j} e^{k_{-} x} \quad \text { for } x \in \mathcal{R}_{j},
$$

where $A_{j}=A_{j}\left(x^{\prime}, s\right)$ and $B_{j}=B_{j}\left(x^{\prime}, s\right)$, with $j=1,2,3$, are arbitrary complex-valued functions and $k_{+}$and $k_{-}$are given by

$$
k_{ \pm}= \pm e^{-i \frac{\pi}{4}} \sqrt{\frac{s}{\alpha}} .
$$

Restricting $s$ to the complex plane branch $-\pi<\arg (s)<\pi$, so that $\operatorname{Re}(\sqrt{s})>0$, we obtain from Eq. (51c)

$$
B_{1}=A_{3}=0 \text {. }
$$

The four remaining conditions, Eqs. (51a), (51b), (51d), and (51e), lead to the matrix equation

$$
\left(\begin{array}{cccc}
-e^{k_{+} x^{\prime}} & e^{k_{+} x^{\prime}} & e^{k_{-}-x^{\prime}} & 0 \\
k_{+} e^{k_{+} x^{\prime}} & k_{+} e^{k_{+} x^{\prime}} & -k_{-} e_{-}^{k_{-} x^{\prime}} & 0 \\
0 & -1 & -1 & 1 \\
0 & -k_{+} & -k_{-} & k_{-}
\end{array}\right)\left(\begin{array}{l}
A_{1} \\
A_{2} \\
B_{2} \\
B_{3}
\end{array}\right)=\left(\begin{array}{c}
0 \\
\frac{i}{\alpha} \\
0 \\
\bar{Q}
\end{array}\right) .
$$

The solution of this matrix equation gives the remaining coefficients

$$
\begin{aligned}
& A_{1}=\frac{e^{-i \frac{\pi}{4}}}{2} \frac{e^{-k_{+} x^{\prime}}}{\sqrt{\alpha s}}-\frac{e^{i \frac{\pi}{4}}}{2} \sqrt{\frac{\alpha}{s}} \bar{Q}(s), \\
& A_{2}=-\frac{e^{i \frac{\pi}{4}}}{2} \sqrt{\frac{\alpha}{s}} \bar{Q}(s), \\
& B_{2}=\frac{e^{-i \frac{\pi}{4}}}{2} \frac{e^{-k_{-} x^{\prime}}}{\sqrt{\alpha s}}, \\
& B_{3}=\frac{e^{-i \frac{\pi}{4}}}{2} \frac{e^{-k_{-} x^{\prime}}}{\sqrt{\alpha s}}-\frac{e^{i \frac{\pi}{4}}}{2} \sqrt{\frac{\alpha}{s}} \bar{Q}(s) .
\end{aligned}
$$

Now we substitute Eqs. (55) and (57) into Eq. (53) and take the inverse Laplace transform on each of the three intervals $\mathcal{R}_{1}, \mathcal{R}_{3}$, and $\mathcal{R}_{3}$. Here we use the fact that $\mathscr{L}^{-1}[\exp (-a \sqrt{s}) / \sqrt{s}]=(\pi \tau)^{-1 / 2} \exp \left(-a^{2} / 4 \tau\right)$ for $\operatorname{Re}(a) \geqslant$ 0 [39] and apply the convolution theorem in computing the inverse Laplace transform of terms of the form $[\exp (-a \sqrt{s}) / \sqrt{s}] \bar{Q}(s)$ with $\operatorname{Re}(a) \geqslant 0$. This computation shows that the propagator $K_{11}\left(x, x^{\prime}, \tau\right)$ has the same expression in all three spatial intervals $\mathcal{R}_{1}, \mathcal{R}_{3}$, and $\mathcal{R}_{3}$, reading

$$
\begin{aligned}
K_{11}\left(x, x^{\prime}, \tau\right)= & K_{0}\left(x-x^{\prime}, \tau\right)+\frac{1}{2} \int_{0}^{\tau} d \tau_{1} \\
& \times \frac{x^{\prime} \kappa\left(x^{\prime}, \tau_{1}\right)}{\tau_{1}} K_{0}\left(x, \tau-\tau_{1}\right) K_{0}\left(x^{\prime}, \tau_{1}\right) .
\end{aligned}
$$

\section{Comparison between the AFM and DPM}

We are now in a position to compare the wave functions $\Psi_{\mathrm{AFM}}(x, t)$ and $\Psi_{\mathrm{DPM}}(x, t)$. First we note that in view of Eqs. (6) and (7) and Eqs. (20) and (58), both wave functions can be written as

$$
\Psi(x, t)=\int_{0}^{t} d \tau \int_{-\infty}^{0} d x^{\prime} F K_{0}(x, t-\tau) K_{0}\left(x^{\prime}, \tau\right) \Psi_{0}\left(x^{\prime}\right),
$$

where $\Psi_{\mathrm{AFM}}$ is obtained by taking $F$ to be

$$
F_{\mathrm{AFM}}\left(x, x^{\prime}, \tau, t\right) \equiv \frac{1}{2} \chi(\tau)\left(\frac{x}{t-\tau}-\frac{x^{\prime}}{\tau}\right)
$$

and $\Psi_{\text {DPM }}$ by taking $F$ to be

$$
\begin{aligned}
F_{\mathrm{DPM}}\left(x, x^{\prime}, \tau, t\right) & \equiv \frac{1}{2}\left[\frac{x}{t-\tau}-\frac{x^{\prime}}{\tau}\left[1-\kappa\left(x^{\prime}, \tau\right)\right]\right] \\
& =\frac{1}{2}\left[\frac{x}{t-\tau}-\frac{x^{\prime}}{\tau}\left(1-\frac{2}{1+\left(\frac{x^{\prime}}{\tau \Omega(\tau)}\right)^{2}}\right)\right] .
\end{aligned}
$$

Here we have used Eq. (47), along with the integral representation of the free-particle propagator

$$
K_{0}\left(x-x^{\prime}, t\right)=\int_{0}^{t} \frac{d \tau}{2}\left(\frac{x}{t-\tau}-\frac{x^{\prime}}{\tau}\right) K_{0}(x, t-\tau) K_{0}\left(x^{\prime}, \tau\right)
$$

obtained from Eq. (7) by taking $\chi(\tau)=1$.

We now show that if the functions $\chi$ and $\Omega$ are related through Eq. (15), then the values of $\Psi_{\mathrm{AFM}}(x, t)$ and $\Psi_{\mathrm{DPM}}(x, t)$ are close in the vicinity of the point $x=x_{t}$, which is the center of the corresponding free-particle wave packet. Indeed, within the semiclassical regime specified by Eq. (16) and for the values of $x$ close to $x_{t}$, the dominant contribution to the double integral in Eq. (59) comes from a neighborhood of the spatial point $x^{\prime}=x_{0}$, around which the amplitude of $\Psi_{0}$ is peaked, and time $\tau=t_{c}$, at which the corresponding classical particle reaches the barrier. Since the barrier, and so the function $F$, is assumed to change in time slowly compared to the exponential terms contained in $K_{0}$ and $\Psi_{0}$, the function $F\left(x, x^{\prime}, \tau, t\right)$ in Eq. (59) can be effectively replaced by $F\left(x_{t}, x_{0}, t_{c}, t\right)$. In the case of the AFM, we have

$$
F_{\mathrm{AFM}}\left(x_{t}, x_{0}, t_{c}, t\right)=v_{0} \chi\left(t_{c}\right),
$$

while in the case of the DPM,

$$
F_{\mathrm{DPM}}\left(x_{t}, x_{0}, t_{c}, t\right)=\frac{v_{0}}{1+\left[\Omega\left(t_{c}\right) / v_{0}\right]^{2}} .
$$

It is now clear that, in view of Eq. (15),

$$
F_{\mathrm{AFM}}\left(x_{t}, x_{0}, t_{c}, t\right)=F_{\mathrm{DPM}}\left(x_{t}, x_{0}, t_{c}, t\right)
$$


and, consequently, $\Psi_{\mathrm{AFM}}(x, t) \simeq \Psi_{\mathrm{DPM}}(x, t)$ around the point $x=x_{t}$.

\section{B. Moshinsky shutter}

We now address a regime opposite to that of a slowly varying barrier. We consider the case in which the barrier stays completely closed during $0<\tau<t_{c}$, opens instantaneously at time $\tau=t_{c}$, and then remains fully open during $t_{c}<\tau<t$. In the context of the AFM, this regime is specified by the aperture function

$$
\chi(\tau)=\Theta\left(\tau-t_{c}\right),
$$

where $\Theta$ denotes the Heaviside step function. In the literature, such an instantaneously opening barrier is commonly referred to as the Moshinsky shutter (see Ref. [7] for Moshinsky's original work). According to Eq. (15), the atom-laser coupling strength $\Omega(\tau)$, corresponding to the aperture function given by Eq. (66), is

$$
\Omega(\tau)= \begin{cases}+\infty, & 0<\tau<t_{c} \\ 0, & t_{c}<\tau<t .\end{cases}
$$

Our aim here is to compare the wave packets $\Psi_{\mathrm{AFM}}(x, t)$ and $\Psi_{\mathrm{DPM}}(x, t)$, specified respectively by Eqs. (66) and (67), in the transmission region $x>0$.

In the AFM case, the wave function can be written as

$$
\begin{aligned}
\Psi_{\mathrm{AFM}}(x, t)= & \int_{-\infty}^{0} d x^{\prime \prime} K_{0}\left(x-x^{\prime \prime}, t-t_{c}\right) \\
& \times \int_{-\infty}^{0} d x^{\prime} K_{0}\left(x^{\prime \prime}-x^{\prime}, t_{c}\right) \Psi_{0}\left(x^{\prime}\right) .
\end{aligned}
$$

The equivalence of this composition-property-type representation of the wave function and the time-integral representation given by Eqs. (6), (7), and (66) has been established in Ref. [19]. Now, within the semiclassical regime specified by Eq. (16), we first evaluate the integral over $x^{\prime}$ and then the other integral over $x^{\prime \prime}$ to find

$$
\Psi_{\mathrm{AFM}}(x, t)=\frac{1}{2} \Psi_{\mathrm{fr}}(x, t)\left[1+\operatorname{erf}\left(e^{i 3 \pi / 4} \sqrt{\frac{m\left(x-x_{t}\right)^{2}}{2 \hbar\left(t-t_{c}\right)}}\right)\right],
$$

where $\operatorname{erf}(\cdot)$ denotes the error function and

$$
\begin{aligned}
\Psi_{\mathrm{fr}}(\xi, \tau)= & \left(\frac{1}{\pi \sigma^{2}}\right)^{1 / 4} \exp \left[-\frac{\left(\xi-x_{\tau}\right)^{2}}{2 \sigma^{2}}\right. \\
& \left.+i \frac{m v_{0}}{\hbar}\left(\xi-x_{\tau}\right)+i \frac{m v_{0}^{2} \tau}{2 \hbar}\right]
\end{aligned}
$$

is the frozen Gaussian (or nondispersive) approximation of the free-particle wave packet defined by Eq. (4).

We now compute the wave function $\Psi_{\mathrm{DPM}}(x, t)$ corresponding to Eq. (67). First, we express the full matrix propagator $\widehat{K}$ as a sequence of two constant potential propagators

$$
\widehat{K}\left(x, x^{\prime}, t\right)=\int_{-\infty}^{+\infty} d x^{\prime \prime} \widehat{K}_{0}\left(x-x^{\prime \prime}, t-t_{c}\right) \widehat{K}_{\infty}\left(x^{\prime \prime}, x^{\prime}, t_{c}\right)
$$

where $\widehat{K}_{0}$ is the matrix free-particle propagator defined by Eq. (26), and

$$
\widehat{K}_{\infty}\left(\xi_{1}, \xi_{2}, \tau\right)=\lim _{\Omega_{0} \rightarrow+\infty} \widehat{K}_{\Omega_{0}}\left(\xi_{1}, \xi_{2}, \tau\right),
$$

where $\widehat{K}_{\Omega_{0}}$ denotes the propagator corresponding to a timeindependent coupling frequency $\Omega(\tau)=\Omega_{0}$. An exact expression for $\widehat{K}_{\Omega_{0}}$ has been derived in Ref. [22] and reads

$$
\begin{aligned}
\widehat{K}_{\Omega_{0}}\left(\xi_{1}, \xi_{2}, \tau\right)= & \widehat{K}_{0}\left(\xi_{1}-\xi_{2}, \tau\right)-\frac{m \Omega_{0}}{4 \hbar} \sum_{j= \pm 1} e^{j \frac{m \Omega_{0}}{\hbar}\left(\left|\xi_{1}\right|+\left|\xi_{2}\right|\right)} \\
& \times e^{i \frac{m \Omega_{0}^{2}}{2 \hbar} \tau} \operatorname{erfc}\left(z_{j}\right)\left(\begin{array}{ll}
j & 1 \\
1 & j
\end{array}\right)
\end{aligned}
$$

with

$$
z_{j}=j \sqrt{i \frac{m \Omega_{0}^{2}}{2 \hbar}} \tau+\sqrt{\frac{m}{2 i \hbar \tau}}\left(\left|\xi_{1}\right|+\left|\xi_{2}\right|\right) .
$$

Here $\operatorname{erfc}(\cdot)=1-\operatorname{erf}(\cdot)$ is the complementary error function.

We readily see from Eq. (74) that $-3 \pi / 4<\arg \left(z_{j}\right)<\pi / 4$ as long as $\xi_{1}, \xi_{2} \neq 0$ and also that $\lim _{\Omega_{0} \rightarrow+\infty}\left|z_{j}\right|=+\infty$. Therefore, using the asymptotic expansion [40] $\operatorname{erfc}\left(z_{j}\right) \simeq$ $\exp \left(-z_{j}^{2}\right) / \sqrt{\pi} z_{j}$ in Eq. (73), substituting the resulting expression into Eq. (72), and taking the limit $\Omega_{0} \rightarrow+\infty$, we obtain

$$
\widehat{K}_{\infty}\left(\xi_{1}, \xi_{2}, \tau\right)=\widehat{K}_{0}\left(\xi_{1}-\xi_{2}, \tau\right)-\widehat{K}_{0}\left(\left|\xi_{1}\right|+\left|\xi_{2}\right|, \tau\right) .
$$

A substitution of Eq. (75) into Eq. (71) yields the following expression for the DPM propagator:

$$
\begin{aligned}
K_{11}\left(x, x^{\prime}, t\right)= & K_{0}\left(x-x^{\prime}, t\right)-\int_{-\infty}^{+\infty} d x^{\prime \prime} \\
& \times K_{0}\left(x-x^{\prime \prime}, t-t_{c}\right) K_{0}\left(\left|x^{\prime}\right|+\left|x^{\prime \prime}\right|, t_{c}\right) .
\end{aligned}
$$

The DPM wave function is then obtained by substituting Eq. (76) into Eq. (20) and evaluating the resulting integrals. So, performing the integration over the initial position $x^{\prime}$ and using the semiclassical limit, we get

$$
\begin{aligned}
\Psi_{\mathrm{DPM}}(x, t)= & \Psi_{\mathrm{fr}}(x, t)-\int_{-\infty}^{+\infty} d x^{\prime \prime} K_{0}\left(x-x^{\prime \prime}, t-t_{c}\right) \\
& \times \Psi_{\mathrm{fr}}\left(\left|x^{\prime \prime}\right|, t_{c}\right) .
\end{aligned}
$$

Finally, calculating the integral in Eq. (77), we establish the following relation between the DPM and AFM wave functions:

$$
\Psi_{\mathrm{DPM}}(x, t)=\Psi_{\mathrm{AFM}}(x, t)+\Delta \Psi(x, t),
$$

where, within the semiclassical regime defined by Eq. (16),

$$
\Delta \Psi(x, t)=\left(\frac{1}{\pi \sigma^{2}}\right)^{1 / 4} \sqrt{\frac{\hbar\left(t-t_{c}\right)}{2 \pi m\left(x+x_{t}\right)^{2}}} e^{i \beta}
$$

with $\beta=m x^{2} / 2 \hbar\left(t-t_{c}\right)+m v_{0}^{2} t_{c} / 2 \hbar-3 \pi / 4$.

It follows from Eqs. (78) and (79) that the values of $\Psi_{\mathrm{AFM}}(x, t)$ and $\Psi_{\mathrm{DPM}}(x, t)$ are close to each other in the vicinity of the spatial point $x=x_{t}$. Indeed, for $x$ close to $x_{t}$, we have

$$
|\Delta \Psi|<\sqrt{\frac{\hbar t}{m x_{t}^{2}}}\left|\Psi_{\mathrm{AFM}}\right| \ll \sqrt{\frac{\hbar t}{m \sigma^{2}}}\left|\Psi_{\mathrm{AFM}}\right| \ll\left|\Psi_{\mathrm{AFM}}\right| .
$$


Far in the tails however, the two wave functions exhibit different behavior. Indeed, an asymptotic expansion of the right-hand side in Eq. (69) shows that $\left|\Psi_{\mathrm{AFM}}\right|^{2} \sim 1 / x^{2}$ as $x \rightarrow$ $\pm \infty$, whereas the corresponding expansion of Eq. (78), with Eqs. (69) and (79) taken into account, yields $\left|\Psi_{\mathrm{DPM}}\right|^{2} \sim 1 / x^{4}$ as $x \rightarrow \pm \infty$.

The analysis presented in this section only establishes agreement between the predictions of the AFM and DPM in a narrow spatial region centered around the point $x=$ $x_{t}$, the point specifying the location of the corresponding freely propagating classical particle at time $t$. In the next section, Sec. IV, we strengthen our statement by numerically demonstrating that the agreement between the two models holds in a much broader spatial region.

\section{NUMERICAL RESULTS}

In this section we numerically evaluate the wave functions $\Psi_{\mathrm{AFM}}(x, t)$ and $\Psi_{\mathrm{DPM}}(x, t)$ and investigate the validity of the relation between $\chi$ and $\Omega$, Eq. (15), in both the semiclassical and deep quantum regimes. We begin by outlining our strategy.

In our numerical study, we focus on two different measures of similarity between the wave functions. The first one, the so-called fidelity, is an overlap between $\Psi_{\mathrm{AFM}}$ and $\Psi_{\mathrm{DPM}}$ on a spatial interval $0<x_{A}<x<x_{B}$ at a fixed time $t>0$. Denoted by $M$, the fidelity is defined as

$$
M(t) \equiv \frac{\left|\int_{x_{A}}^{x_{B}} d x \Psi_{\mathrm{AFM}}^{\star}(x, t) \Psi_{\mathrm{DPM}}(x, t)\right|^{2}}{\mathscr{P}_{\mathrm{AFM}}(t) \mathscr{P}_{\mathrm{DPM}}(t)},
$$

where

$$
\mathscr{P}_{\mathrm{AFM}, \mathrm{DPM}}(t) \equiv \int_{x_{A}}^{x_{B}} d x\left|\Psi_{\mathrm{AFM}, \mathrm{DPM}}(x, t)\right|^{2}
$$

represents the probability of finding the particle inside the interval $x_{A}<x<x_{B}$ at time $t$, as predicted by the AFM or DPM, respectively. The interval boundaries $x_{A}$ and $x_{B}$ are numerical parameters and will be further chosen such that the interval contains the dominant part of the transmitted wave function. By construction, the fidelity takes values between 0 and 1 . Here $M(t)=0$ means that the two wave functions are mutually orthogonal at time $t$ and so completely different from one another. On the other hand, $M(t)=1$ is reached whenever the functional form of $\Psi_{\mathrm{AFM}}$ is identical to that of $\Psi_{\mathrm{DPM}}$ up to an arbitrary normalization constant.

Since the fidelity, as defined by Eq. (81), is insensitive to the global amplitudes of $\Psi_{\mathrm{AFM}}$ and $\Psi_{\mathrm{DPM}}$, we use the probability ratio

$$
R(t) \equiv \frac{\mathscr{P}_{\mathrm{DPM}}(t)}{\mathscr{P}_{\mathrm{AFM}}(t)}
$$

as our second tool to compare the AFM and DPM wave functions. It is worth nothing that $R$ merely compares the overall probabilities of finding the particle inside the region $x_{A}<x<x_{B}$ at time $t$ as predicted by the AFM and the DPM and thus complements the fidelity test.

In this section we numerically evaluate the fidelity $M(t)$ and probability ratio $R(t)$ in four atom-barrier systems that differ from each other only by the mass of the atom. More specifically, we consider the dynamics of the alkali-metal atoms ${ }^{7} \mathrm{Li},{ }^{23} \mathrm{Na},{ }^{41} \mathrm{~K}$, and ${ }^{87} \mathrm{Rb}$, which are routinely used in modern ultracold atom-optics experiments. The atomic masses are $m_{\mathrm{Li}}=7.016003 \mathrm{u}, m_{\mathrm{Na}}=22.989767 \mathrm{u}, m_{\mathrm{K}}=$ $40.961825 \mathrm{u}$, and $m_{\mathrm{Rb}}=86.9091805 \mathrm{u}$, respectively. Other parameters are the same for all four systems and have the following values. The initial wave packet, defined by Eq. (13), is characterized by the initial position (with respect to the position of the barrier) $x_{0}=-0.15 \mathrm{~mm}$, spatial dispersion $\sigma=30 \mu \mathrm{m}$, and average velocity $v_{0}=3 \mathrm{~mm} / \mathrm{s}$. The total propagation time is taken to be $t=100 \mathrm{~ms}$. These parameter values imply the classical barrier crossing time $t_{c}=50 \mathrm{~ms}=$ $t / 2$ and the final position of the unperturbed classical particle $x_{t}=0.15 \mathrm{~mm}=\left|x_{0}\right|$. We note that the chosen parameter values are comparable to values in real laboratory experiments [33-35].

It can be easily seen that while the parameters of the heaviest (rubidium) system satisfy the semiclassical regime conditions, given by Eq. (16), the parameters of the lightest (lithium) system do not. Indeed, in the case of ${ }^{87} \mathrm{Rb}$, we have $m_{\mathrm{Rb}} \sigma^{2} v_{0} / \hbar \simeq 3.7 \mathrm{~mm}$, which is more than 20 times larger than $v_{0}\left(t-t_{c}\right)=0.15 \mathrm{~mm}$; on the other hand, in the case of ${ }^{7} \mathrm{Li}$, we have $m_{\mathrm{Li}} \sigma^{2} v_{0} / \hbar \simeq 0.3 \mathrm{~mm}$, which is comparable to $v_{0}\left(t-t_{c}\right)=0.15 \mathrm{~mm}$. Thus, by decreasing the mass of the moving particle from $m_{\mathrm{Rb}}$ to $m_{\mathrm{Li}}$ we can test the agreement between the AFM and DPM both within and outside the semiclassical regime.

Now, having specified the numerical values of all system parameters and equipped with Eq. (15), we evaluate and compare the wave functions $\Psi_{\mathrm{AFM}}$ and $\Psi_{\mathrm{DPM}}$ in four different scenarios. First, we consider the case of a time-independent barrier given by $\Omega(\tau)=\Omega_{0}$ and $\chi(\tau)=\chi_{0}=\left[1+\left(\Omega_{0} / v_{0}\right)^{2}\right]^{-1}$. Second, we address the exactly solvable case of a slowly (algebraically) varying barrier characterized by $\Omega(\tau)=\Omega_{1} / \tau$. Third, we address barriers whose transparency changes exponentially in time. Finally, we take a closer look at the instantaneous shutter case, previously discussed in Sec. III B.

\section{A. Time-independent barrier}

We begin by considering the simplest scenario in which $\chi(\tau)=\chi_{0}$ and $\Omega(\tau)=\Omega_{0}$, where $\chi_{0}$ and $\Omega_{0}$ are constants related to each other by Eq. (15), i.e., $\chi_{0}=\left[1+\left(\Omega_{0} / v_{0}\right)^{2}\right]^{-1}$. In this case, the AFM wave function is simply an attenuated free-particle Gaussian wave packet $\Psi_{\mathrm{AFM}}=\chi_{0} \Psi_{\text {free }}$, with $\Psi_{\text {free }}$ defined by Eq. (4). The DPM wave function can be obtained from Eq. (20) by taking $K_{11}=\left(\widehat{K}_{\Omega_{0}}\right)_{11}$, where $\widehat{K}_{\Omega_{0}}$ is the exact DPM propagator given by Eq. (73).

Our definitions of the fidelity and probability ratio, given by Eqs. (81) and (83), respectively, depend on the integration region $x_{A}<x<x_{B}$. Here we choose $x_{A}=x_{t}-4(\Delta x)_{t}$ and $x_{B}=x_{t}+4(\Delta x)_{t}$, where $(\Delta x)_{t}=(\sigma / \sqrt{2}) \sqrt{1+\left(\hbar t / m \sigma^{2}\right)^{2}}$ is the position uncertainty of the free-particle wave packet $\Psi_{\text {free }}$ at time $t$. This choice guarantees that the comparison between the AFM and DPM wave functions is performed on a very broad spatial interval centered around the classically expected position of the particle. For the parameter values specified above, we have $(\Delta x)_{t} \simeq 30.1 \mu \mathrm{m}$ for ${ }^{7} \mathrm{Li}, 22.2 \mu \mathrm{m}$ for ${ }^{23} \mathrm{Na}, 21.5 \mu \mathrm{m}$ for ${ }^{41} \mathrm{~K}$, and $21.3 \mu \mathrm{m}$ for ${ }^{87} \mathrm{Rb}$.

Figure 1(a) shows the values of the fidelity and probability ratio deviations from $1,1-M$, and $|1-R|$, respectively, maximized over the wide range of barrier strengths 

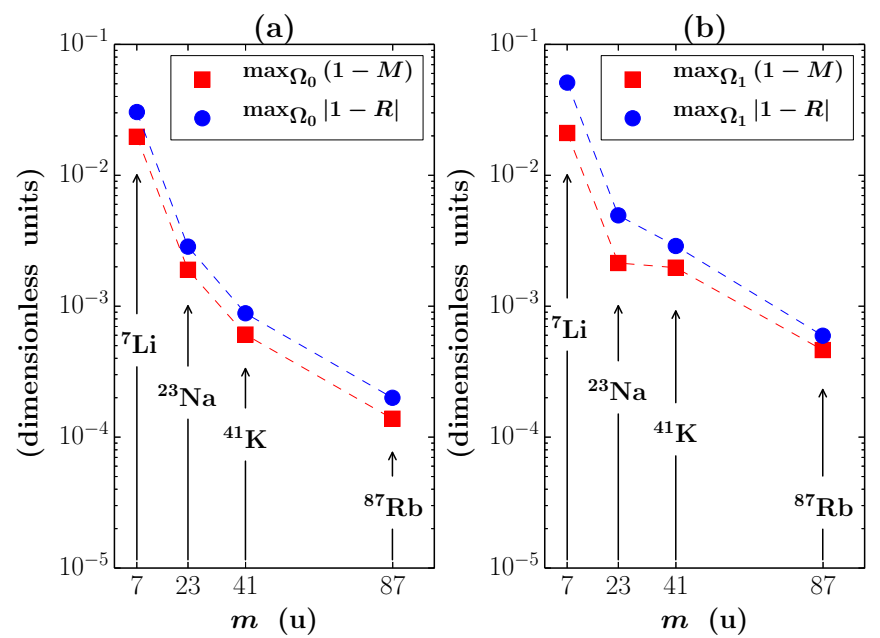

FIG. 1. (Color online) Maximal deviations of the fidelity $M$ (red squares) and probability ratio $R$ (blue circles) from 1 for four different alkali-metal atoms. (a) Data represent the case of a time-independent barrier with $\Omega(\tau)=\Omega_{0}$; the maximal deviations are computed with respect to $\Omega_{0}$. (b) Data correspond to $\Omega(\tau)=\Omega_{1} / \tau$; the maximal deviations are computed with respect to $\Omega_{1}$. See the text for all parameter values.

$0 \leqslant \Omega_{0} \leqslant 100 v_{0}$. We clearly see that the agreement between $\Psi_{\mathrm{AFM}}$ and $\Psi_{\mathrm{DPM}}$ significantly improves- $\max _{\Omega_{0}}(1-M)$ and $\max _{\Omega_{0}}|1-R|$ decrease by over two orders of magnitude-as the atomic mass increases from $m_{\mathrm{Li}}$ to $m_{\mathrm{Rb}}$. On a more practical side, in the cases of potassium and rubidium, the wave functions predicted by the AFM and DPM appear to be almost indistinguishable: Already for potassium, $M$ and $R$ deviate from 1 by less than $0.1 \%$.

\section{B. Algebraic barrier}

As our first example of a time-dependent barrier, we consider the scenario in which the atom-laser interaction is inversely proportional to time, i.e., $\Omega(\tau)=\Omega_{1} / \tau$. The DPM in this case is exactly solvable. Indeed, introducing $\psi_{ \pm}=$ $\psi_{1} \pm \psi_{2}$ one rewrites Eq. (8) as two uncoupled Schrödinger equations for a single-channel potential of the form $\tau^{-1} \delta(x)$. The latter problem has been solved in Ref. [32]. Using this solution, we obtain the following expression for the full propagator of the DPM:

$$
\begin{aligned}
\widehat{K}\left(x, x^{\prime}, \tau\right)= & \widehat{K}_{0}\left(x-x^{\prime}, \tau\right)-\frac{\Omega_{1}}{\Omega_{1}^{2}+x^{\prime 2}} K_{0}\left(|x|+\left|x^{\prime}\right|, \tau\right) \\
& \times\left(\begin{array}{cc}
\Omega_{1} & i\left|x^{\prime}\right| \\
i\left|x^{\prime}\right| & \Omega_{1}
\end{array}\right) .
\end{aligned}
$$

We compute the DPM wave function by substituting the first element of the matrix propagator $\widehat{K}$,

$$
K_{11}\left(x, x^{\prime}, \tau\right)=K_{0}\left(x-x^{\prime}, \tau\right)-\frac{\Omega_{1}^{2}}{\Omega_{1}^{2}+x^{\prime 2}} K_{0}\left(|x|+\left|x^{\prime}\right|, \tau\right),
$$

into Eq. (20) and evaluating the resulting integral numerically. The corresponding AFM wave function is obtained from Eqs. (6) and (7) by taking $\chi(\tau)=\left[1+\left(\Omega_{1} / v_{0} \tau\right)^{2}\right]^{-1}$, in accord with Eq. (15). As in the time-independent case of
Sec. IV A, the fidelity and probability ratio are computed by taking $x_{A}=x_{t}-4(\Delta x)_{t}$ and $x_{B}=x_{t}+4(\Delta x)_{t}$.

Figure 1(b) shows $\max _{\Omega_{1}}(1-M)$ (red squares) and $\max _{\Omega_{1}}|1-R|$ (blue circles) corresponding to the range $0 \leqslant$ $\Omega_{1} \leqslant 100 v_{0} t_{c}$. Once again, we observe the agreement between the predictions of the AFM and DPM improve as the mass of the atom increases. In particular, in the case of rubidium, the fidelity and probability ratio deviate from 1 by less than $0.1 \%$.

\section{Exponential barriers}

We now consider absorbing barriers whose aperture function $\chi(\tau)$ exhibits exponential dependence on time on some intervals. As recently shown in Ref. [21], such barriers can be efficiently used to manipulate, e.g., shift, split, or squeeze, the spatial wave function of the transmitted particle. Here we consider three different scenarios defined by the aperture functions presented in Figs. 2(a)-2(c). The corresponding atom-laser interaction strengths are computed using Eq. (15) and shown in Figs. 2(d)-2(f). The AFM wave function is calculated by evaluating the integrals in Eqs. (6) and (7). The DPM wave function is obtained by solving the time-dependent Schrödinger equation [Eqs. (8)-(11)] numerically, using a Crank-Nicolson algorithm [41].

Figure 3 shows the probability densities $\left|\Psi_{\mathrm{AFM}}(x, t)\right|^{2}$ (solid curves) and $\left|\Psi_{\mathrm{DPM}}(x, t)\right|^{2}$ (dash-dotted curves) for all four atoms as functions of $x$. Blue curves correspond to the barrier defined by $\chi(\tau)=\min \left\{\chi_{>}(\tau), 1\right\}$, with $\chi_{>}(\tau)=$ $\exp \left[\gamma\left(\tau-3 t_{c} / 2\right)\right]$ and $\gamma=100 \mathrm{~s}^{-1}$ [see Figs. 2(a) and 2(d)]. Red curves correspond to the barrier defined by $\chi(\tau)=\min \left\{\chi_{<}(\tau), 1\right\}$, with $\chi_{<}(\tau)=\exp \left[\gamma\left(\tau-t_{c} / 2\right)\right]$ and $\gamma=-100 \mathrm{~s}^{-1}$ [see Figs. 2(b) and 2(e)]. The dotted green curve
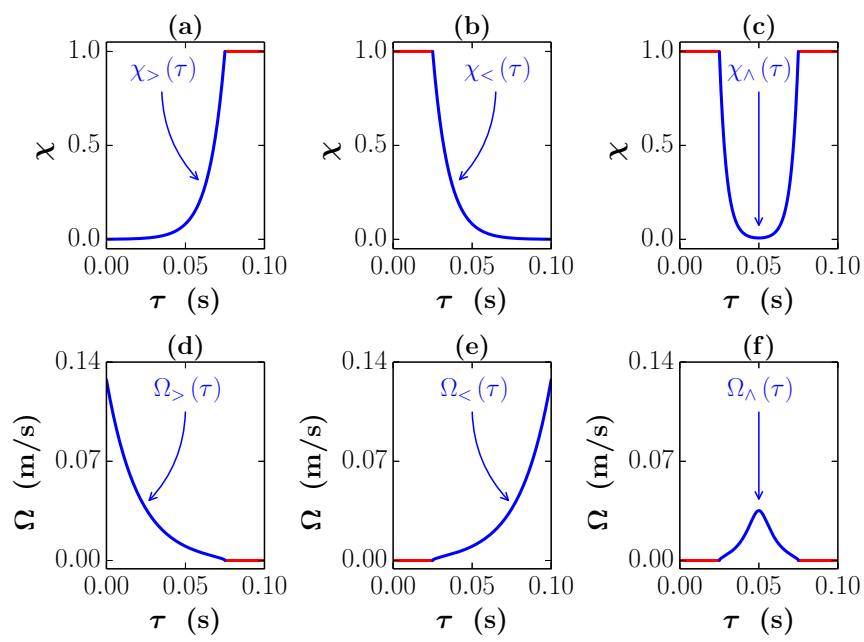

FIG. 2. (Color online) Aperture function $\chi(\tau)$ and the corresponding atom-laser interaction strength $\Omega(\tau)$ in three different scenarios: (a) $\chi(\tau)=\min \left\{\chi_{>}(\tau), 1\right\}$, with $\chi_{>}(\tau) \equiv \exp [\gamma(\tau-$ $\left.\left.3 t_{c} / 2\right)\right]$ and $\gamma=100 \mathrm{~s}^{-1}$; (b) $\chi(\tau)=\min \left\{\chi_{<}(\tau), 1\right\}$, with $\chi_{<}(\tau) \equiv$ $\exp \left[\gamma\left(\tau-t_{c} / 2\right)\right]$ and $\gamma=-100 \mathrm{~s}^{-1} ;$ (c) $\chi(\tau)=\min \left\{\chi_{\wedge}(\tau), 1\right\}$, with $\chi_{\wedge}(\tau) \equiv \cosh \left[\gamma\left(\tau-t_{c}\right)\right] / \cosh \left(\gamma t_{c} / 2\right)$ and $\gamma=225 \mathrm{~s}^{-1}$; (d) $\Omega(\tau)=\Omega_{>}(\tau) \equiv v_{0} \sqrt{1 / \chi_{>}(\tau)-1}$ for $\tau<3 t_{c} / 2$ and $\Omega(\tau)=$ 0 for $\tau \geqslant 3 t_{c} / 2$; (e) $\Omega(\tau)=0$ for $\tau \leqslant t_{c} / 2$ and $\Omega(\tau)=$ $\Omega_{<}(\tau) \equiv v_{0} \sqrt{1 / \chi_{<}(\tau)-1}$ for $\tau>t_{c} / 2$; and (f) $\Omega(\tau)=\Omega_{\wedge}(\tau) \equiv$ $v_{0} \sqrt{1 / \chi_{\wedge}(\tau)-1}$ for $\left|\tau-t_{c}\right|<t_{c} / 2$ and $\Omega(\tau)=0$ for $\left|\tau-t_{c}\right| \geqslant$ $t_{c} / 2$. 

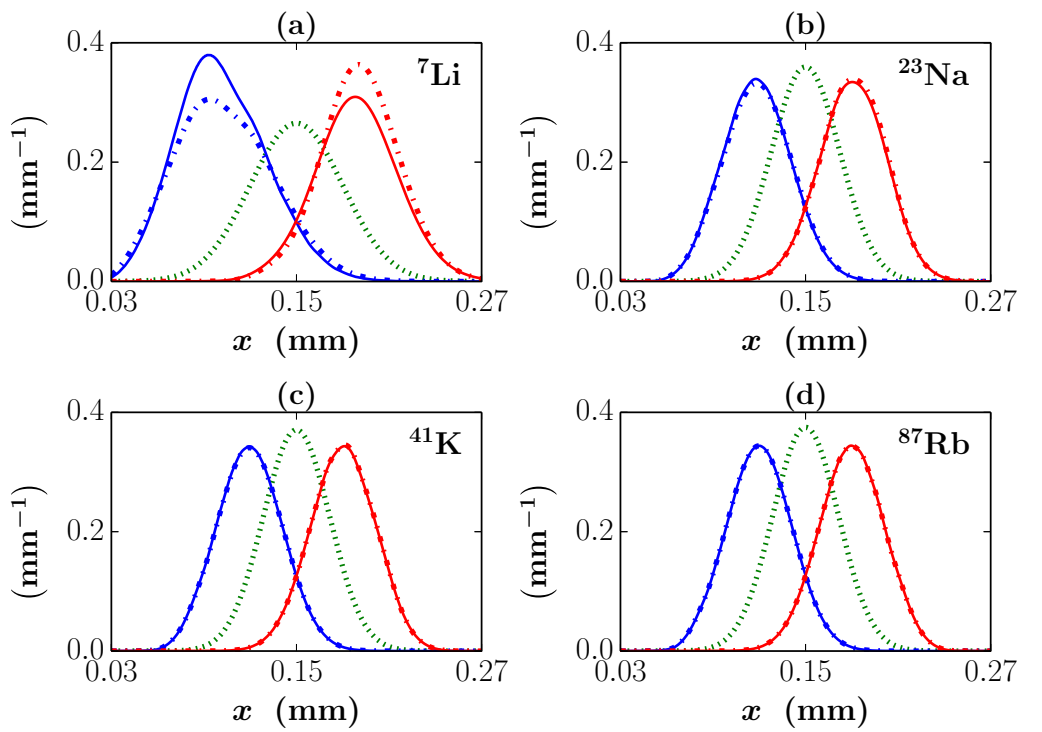

$$
\begin{array}{ll}
\ldots \ldots+1 & \begin{array}{l}
0.02\left|\Psi_{\text {free }}\right|^{2} \\
(\gamma=0)
\end{array} \\
-\quad & \left|\Psi_{\mathrm{AFM}}\right|^{2} \\
\left(\gamma=100 \mathrm{~s}^{-1}\right) \\
\cdots \quad & \left|\Psi_{\mathrm{DPM}}\right|^{2} \\
& \left(\gamma=100 \mathrm{~s}^{-1}\right) \\
-\quad & \left|\Psi_{\mathrm{AFM}}\right|^{2} \\
& \left(\gamma=-100 \mathrm{~s}^{-1}\right) \\
\because, \quad & \left|\Psi_{\mathrm{DPM}}\right|^{2} \\
& \left(\gamma=-100 \mathrm{~s}^{-1}\right)
\end{array}
$$

FIG. 3. (Color online) Probability densities $\left|\Psi_{\mathrm{AFM}}\right|^{2}$ (solid curves) and $\left|\Psi_{\mathrm{DPM}}\right|^{2}$ (dash-dotted curves) for (a) ${ }^{7} \mathrm{Li}$, (b) ${ }^{23} \mathrm{Na}$, (c) ${ }^{41} \mathrm{~K}$, and (d) ${ }^{87} \mathrm{Rb}$ as functions of the position $x$. Blue curves correspond to the barrier specified in Figs. 2(a) and 2(d). Red curves correspond to the barrier specified in Figs. 2(b) and 2(e). The dotted green curve represents the scaled probability density of the corresponding free-particle wave packet.

represents the scaled probability density of the free-particle Gaussian wave packet $0.02\left|\Psi_{\text {free }}(x, t)\right|^{2}$ that would be observed in the absence of a barrier, i.e., for $\gamma=0$. The effect of the barrier is to shift the transmitted wave packet by the distance $\Delta \simeq-\gamma \sigma^{2} / v_{0}$ with respect to the position of the freely evolved Gaussian wave packet [21].

Figure 4 shows the probability densities predicted by the AFM (solid red curve) and DPM (dash-dotted blue curve) for the barrier defined by $\chi(\tau)=\min \left\{\chi_{\wedge}(\tau), 1\right\}$, with $\chi_{\wedge}(\tau) \equiv \cosh \left[\gamma\left(\tau-t_{c}\right)\right] / \cosh \left(\gamma t_{c} / 2\right)$ and $\gamma=225 \mathrm{~s}^{-1}$ [see Figs. 2(c) and 2(f)]. For reference, the dotted green curve shows the scaled probability density of the free-particle Gaussian wave packet $0.003\left|\Psi_{\text {free }}(x, t)\right|^{2}$. The effect of the barrier is to spatially split the transmitted wave packet in two as compared to the freely evolved Gaussian wave packet.

As in the examples considered in Secs. IV A and IV B, we observe in Figs. 3 and 4 that the agreement between the AFM and DPM quickly improves as the atomic mass is increased. More quantitatively, the values of $1-M$ and $|1-R|$ decrease by approximately two orders of magnitude as we go from the case of lithium to that of rubidium.

\section{Moshinsky shutter}

We conclude this section by considering the case of the Moshinsky shutter, i.e., an instantaneously opening barrier with the aperture function defined by Eq. (66). As in the
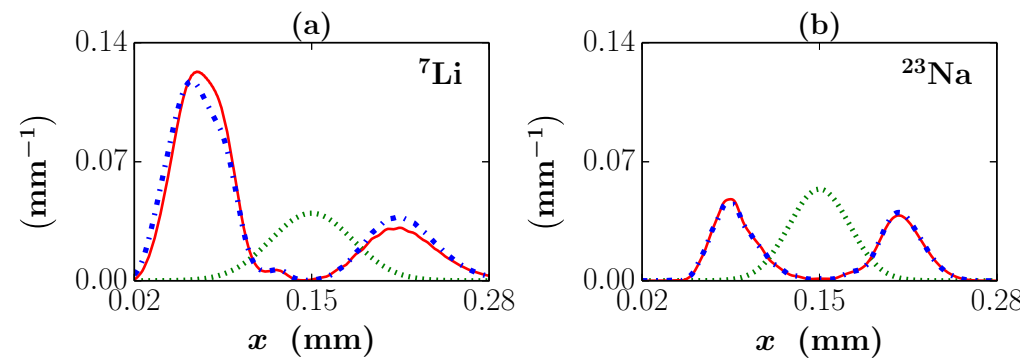

$$
\ldots \begin{aligned}
& 0.003\left|\Psi_{\text {free }}\right|^{2} \\
& (\gamma=0) \\
& -\quad\left|\Psi_{\mathrm{AFM}}\right|^{2}
\end{aligned}
$$
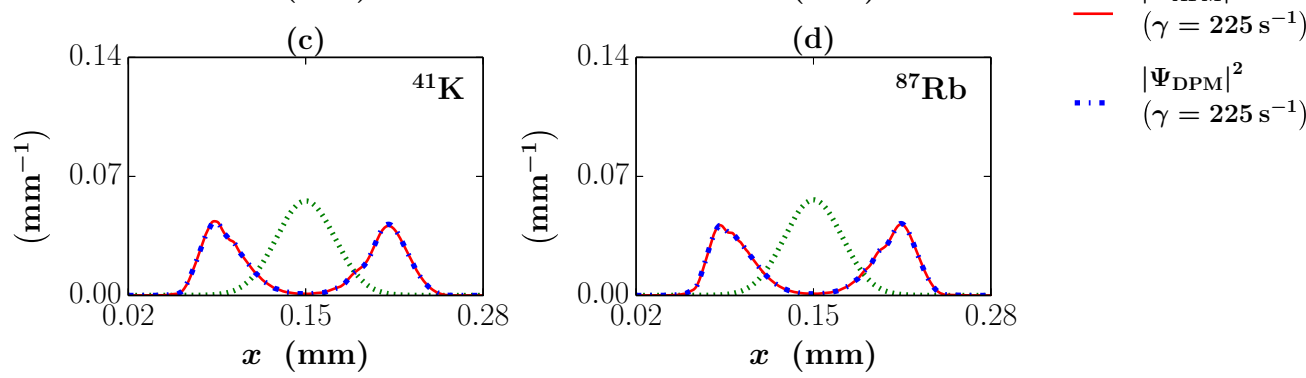

FIG. 4. (Color online) Probability densities $\left|\Psi_{\mathrm{AFM}}\right|^{2}$ (solid red curve) and $\left|\Psi_{\mathrm{DPM}}\right|^{2}$ (dash-dotted blue curve) for (a) ${ }^{7} \mathrm{Li}$, (b) ${ }^{23} \mathrm{Na}$, (c) ${ }^{41} \mathrm{~K}$, and (d) ${ }^{87} \mathrm{Rb}$ as functions of the position $x$. The curves correspond to the barrier specified in Figs. 2(c) and 2(f). The dotted green curve represents the scaled probability density of the corresponding free-particle wave packet. 
(a)

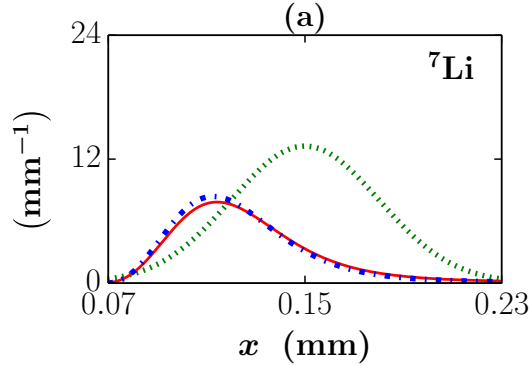

(c)

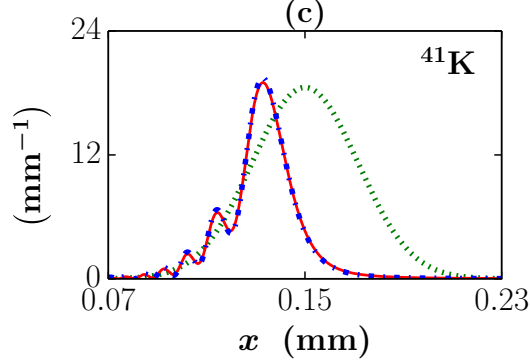

(b)

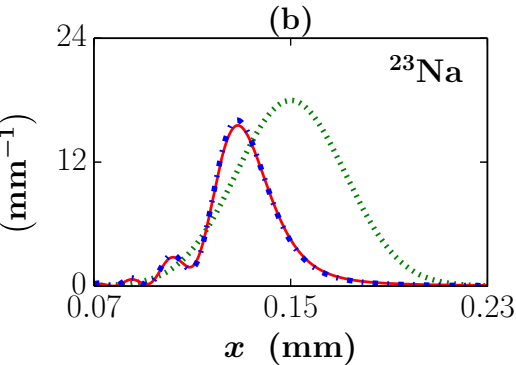

(d)

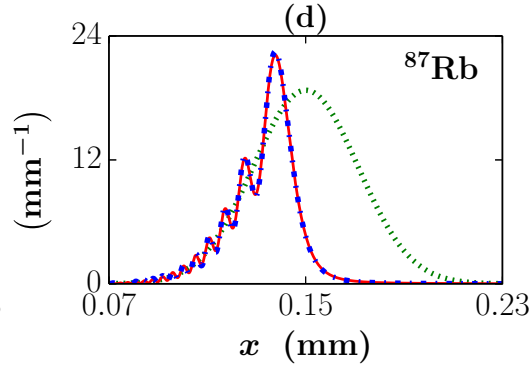

…... $\left|\Psi_{\text {free }}\right|^{2}$

$-\left|\Psi_{\mathrm{AFM}}\right|^{2}$

$\ldots\left|\Psi_{\mathrm{DPM}}\right|^{2}$

FIG. 5. (Color online) Probability densities $\left|\Psi_{\mathrm{AFM}}(x, t)\right|^{2}$ (solid red curve) and $\left|\Psi_{\mathrm{DPM}}(x, t)\right|^{2}$ (dash-dotted blue curve) for (a) ${ }^{7} \mathrm{Li}$, (b) ${ }^{23} \mathrm{Na}$, (c) ${ }^{41} \mathrm{~K}$, and (d) ${ }^{87} \mathrm{Rb}$ evaluated for the case of the Moshinsky shutter [Eq. (66)]. The dotted green curve represents the probability density of the corresponding free-particle wave packet.

previous examples, $\Psi_{\mathrm{AFM}}$ is calculated by using Eqs. (6) and (7), whereas $\Psi_{\text {DPM }}$ is obtained from Eq. (20) with the propagator $K_{11}$ given by Eq. (76).

Figure 5 shows the probability densities $\left|\Psi_{\mathrm{AFM}}\right|^{2}$ (solid red curve), $\left|\Psi_{\text {DPM }}\right|^{2}$ (dash-dotted blue curve), and $\left|\Psi_{\text {free }}\right|^{2}$ (dotted green curve) as functions of the position $x$. The Moshinsky barrier induces oscillations of the probability density of the transmitted wave packet as compared to that of a freely evolved Gaussian. These oscillations get more and more pronounced as the mass of the atom increases.

In accord with all other examples of this section, we find better agreement between the predictions of the AFM and DPM for heavier (more semiclassical) atoms. In particular, the deviation of the fidelity from $1,1-M$, decreases by approximately 14 times as we go from the lithium to the rubidium system; the deviation of the probability ratio $|1-R|$ decreases by approximately 17 times.

\section{CONCLUSION}

In this paper we compared two different theoretical descriptions of the motion of a quantum particle, e.g., an atom, through a time-dependent absorbing pointlike barrier, e.g., a sheet of laser light with a time-dependent intensity. The first one, the aperture function model, represents the barrier by a set of time-dependent discontinuous matching conditions of Kottler type (2) and (3). The key ingredient of the model is a time-dependent transmission amplitude $\chi(\tau)$, entering the matching conditions. The main advantage of the AFM is that it allows for an explicit integral expression of the quantum propagator (7). The second description, the $\delta$-potential model, represents the barrier by an off-diagonal $\delta$ potential with a time-dependent amplitude $\Omega(\tau)$ [Eq. (11)]. The DPM is a time-dependent generalization of the stationary atom-laser interaction model addressed in Ref. [22]. To date, only a few exactly solvable cases of the DPM are known, examples being the systems characterized by $\Omega(\tau)=$ const and $\Omega(\tau)=$ const $/ \tau$. In general, the DPM has to be solved by numerically integrating the time-dependent Schrödinger equation [Eqs. (8)-(11)].

Here we have found a way of mapping the two models onto one another in the semiclassical regime. More concretely, we have shown that, in the transmission region, the wave functions predicted by the AFM and DPM are in good agreement with each other provided that the aperture function $\chi$ and the amplitude $\Omega$ are related through Eq. (15). The agreement improves as the mass of the moving particle increases, i.e., as the system becomes more semiclassical. Our conclusion is based on both asymptotic analytical calculations, presented in Sec. III, and a detailed numerical investigation of four particle-barrier systems with experimentally realistic parameters, reported in Sec. IV.

Time-dependent absorbing barriers may be realized in atom-optics laboratory experiments. Recently, such barriers were identified as a promising tool for engineering and reshaping (e.g., shifting, splitting, squeezing, and cooling) of atomic wave packets [21]. The main practical value of the present study is that we have extended the range of theoretical tools appropriate for investigating and making quantitative predictions related to light-based manipulation of atomic wave functions. In the future, it would be interesting to construct and explore other representations of time-dependent pointlike absorbing barriers, some promising candidates being the imaginary $\delta$ potential [42], and generalized point interactions of the form $c_{1} \delta(x)+c_{2} \delta^{\prime}(x)[43,44]$.

\section{ACKNOWLEDGMENTS}

The authors are thankful to Adolfo del Campo for useful suggestions. A.G. acknowledges financial support from EPSRC under Grant No. EP/K024116/1. M. Beau acknowledges funding support from ESF (POLATOM-5052). 
APPENDIX: DERIVATION OF EQ. (42)

We start by rewriting the Hessian, given by Eq. (41), in the matrix form

$$
\mathcal{H}=2\left(\sum_{k=1}^{n+1}\left|\epsilon_{k}-\epsilon_{k-1}\right|\right)^{3} \mathcal{A}
$$

where

$$
A=\left(\begin{array}{cccccc}
b_{1} & c_{1} & 0 & \ldots & \ldots & 0 \\
a_{2} & \ddots & \ddots & \ddots & & \vdots \\
0 & \ddots & \ddots & \ddots & \ddots & \vdots \\
\vdots & \ddots & \ddots & \ddots & \ddots & 0 \\
\vdots & & \ddots & \ddots & \ddots & c_{n-1} \\
0 & \ldots & \ldots & 0 & a_{n} & b_{n}
\end{array}\right)
$$

with

$$
\begin{aligned}
& a_{j}=-\frac{1}{\left|\epsilon_{j}-\epsilon_{j-1}\right|}, \quad 2 \leqslant j \leqslant n \\
& b_{j}=\frac{1}{\left|\epsilon_{j}-\epsilon_{j-1}\right|}+\frac{1}{\left|\epsilon_{j+1}-\epsilon_{j}\right|}, \quad 1 \leqslant j \leqslant n
\end{aligned}
$$

and

$$
c_{j}=-\frac{1}{\left|\epsilon_{j+1}-\epsilon_{j}\right|}, \quad 1 \leqslant j \leqslant n-1 .
$$

It follows immediately from Eq. (A1) that

$$
\operatorname{det}(\mathcal{H})=2^{n}\left(\sum_{k=1}^{n+1}\left|\epsilon_{k}-\epsilon_{k-1}\right|\right)^{3 n} \operatorname{det}(\mathcal{A})
$$

In order to find $\operatorname{det}(\mathcal{A})$ we use an $L U$ decomposition of the matrix $\mathcal{A}$. That is, we express $\mathcal{A}$ as

$$
\mathcal{A}=\mathcal{L U}
$$

where

$$
\mathcal{L}=\left(\begin{array}{cccccc}
1 & 0 & 0 & \cdots & \cdots & 0 \\
L_{2} & \ddots & \ddots & \ddots & & \vdots \\
0 & \ddots & \ddots & \ddots & \ddots & \vdots \\
\vdots & \ddots & \ddots & \ddots & \ddots & 0 \\
\vdots & & \ddots & \ddots & \ddots & 0 \\
0 & \cdots & \cdots & 0 & L_{n} & 1
\end{array}\right)
$$

and

$$
\mathcal{U}=\left(\begin{array}{cccccc}
U_{1} & c_{1} & 0 & \cdots & \cdots & 0 \\
0 & \ddots & \ddots & \ddots & & \vdots \\
0 & \ddots & \ddots & \ddots & \ddots & \vdots \\
\vdots & \ddots & \ddots & \ddots & \ddots & 0 \\
\vdots & & \ddots & \ddots & \ddots & c_{n-1} \\
0 & \ldots & \cdots & 0 & 0 & U_{n}
\end{array}\right) .
$$

Substituting Eqs. (A2), (A6), and (A7) into Eq. (A5), we see that the $2 n-1$ matrix elements $L_{j}$ and $U_{j}$ must satisfy the following $2 n-1$ equations:

$$
\begin{aligned}
U_{1} & =b_{1}, \\
L_{j+1} U_{j} & =a_{j+1}, \quad 1 \leqslant j \leqslant n-1 \\
L_{j+1} c_{j}+U_{j+1} & =b_{j+1}, \quad 1 \leqslant j \leqslant n-1 .
\end{aligned}
$$

Solving this system of equations, we find

$$
L_{j}=-\frac{\sum_{k=1}^{j-1}\left|\epsilon_{k}-\epsilon_{k-1}\right|}{\sum_{k=1}^{j}\left|\epsilon_{k}-\epsilon_{k-1}\right|}
$$

for all $2 \leqslant j \leqslant n$ and

$$
U_{j}=\frac{\sum_{k=1}^{j+1}\left|\epsilon_{k}-\epsilon_{k-1}\right|}{\left|\epsilon_{j+1}-\epsilon_{j}\right| \sum_{k=1}^{j}\left|\epsilon_{k}-\epsilon_{k-1}\right|}
$$

for all $1 \leqslant j \leqslant n$.

It is now straightforward to compute the determinant of $\mathcal{A}$. In view of Eqs. (A5)-(A7), we have

$$
\operatorname{det}(\mathcal{A})=\operatorname{det}(\mathcal{L}) \operatorname{det}(\mathcal{U})=\prod_{j=1}^{n} U_{j}
$$

and thus, using Eq. (A10),

$$
\operatorname{det}(\mathcal{A})=\frac{\sum_{k=1}^{n+1}\left|\epsilon_{k}-\epsilon_{k-1}\right|}{\prod_{k=1}^{n+1}\left|\epsilon_{k}-\epsilon_{k-1}\right|}
$$

Finally, combining Eqs. (A4) and (A12), we arrive at the final result, Eq. (42).
[1] J. G. Muga, J. P. Palao, B. Navarro, and I. L. Egusquiza, Complex absorbing potentials, Phys. Rep. 395, 357 (2004).

[2] G. R. Hadley, Transparent boundary condition for beam propagation, Opt. Lett. 16, 624 (1991).

[3] T. Shibata, Absorbing boundary conditions for the finitedifference time-domain calculation of the one-dimensional Schrödinger equation, Phys. Rev. B 43, 6760 (1991).

[4] J.-P. Kuska, Absorbing boundary conditions for the Schrödinger equation on finite intervals, Phys. Rev. B 46, 5000 (1992).
[5] G. Pang, L. Bian, and S. Tang, Almost exact boundary condition for one-dimensional Schrödinger equations, Phys. Rev. E 86, 066709 (2012).

[6] F. L. Traversa, Z. Zhan, and X. Oriols, Absorption and injection models for open time-dependent quantum systems, Phys. Rev. E 90, 023304 (2014).

[7] M. Moshinsky, Diffraction in time, Phys. Rev. 88, 625 (1952).

[8] M. Moshinsky, Diffraction in time and the time-energy uncertainty relation, Am. J. Phys. 44, 1037 (1976). 
[9] V. Man'ko, M. Moshinsky, and A. Sharma, Diffraction in time in terms of Wigner distributions and tomographic probabilities, Phys. Rev. A 59, 1809 (1999).

[10] M. Kleber, Exact solutions for time-dependent phenomena in quantum mechanics, Phys. Rep. 236, 331 (1994).

[11] Time in Quantum Mechanics - Vol. 1, edited by J. G. Muga, R. S. Mayato, and I. L. Egusquiza, Lecture Notes in Physics Vol. 734 (Springer, Berlin, 2008).

[12] Time in Quantum Mechanics_Vol. 2, edited by J. G. Muga, A. Ruschhaupt, and A. del Campo, Lecture Notes in Physics Vol. 789 (Springer, Berlin, 2009).

[13] A. del Campo, G. García-Calderón, and J. G. Muga, Quantum transients, Phys. Rep. 476, 1 (2009).

[14] P. Szriftgiser, D. Guéry-Odelin, M. Arndt, and J. Dalibard, Atomic Wave Diffraction and Interference Using Temporal Slits, Phys. Rev. Lett. 77, 4 (1996).

[15] T. Hils, J. Felber, R. Gähler, W. Gläser, R. Golub, K. Habicht, and P. Wille, Matter-wave optics in the time domain: Results of a cold-neutron experiment, Phys. Rev. A 58, 4784 (1998).

[16] F. Lindner, M. G. Schätzel, H. Walther, A. Baltuška, E. Goulielmakis, F. Krausz, D. B. Milošević, D. Bauer, W. Becker, and G. G. Paulus, Attosecond Double-Slit Experiment, Phys. Rev. Lett. 95, 040401 (2005).

[17] Y. Colombe, B. Mercier, H. Perrin, and V. Lorent, Diffraction of a Bose-Einstein condensate in the time domain, Phys. Rev. A 72, 061601(R) (2005).

[18] G. G. Paulus and D. Bauer, in Time in Quantum MechanicsVol. 2 (Ref. [12]), Chap. 11, pp. 303-339.

[19] A. Goussev, Huygens-Fresnel-Kirchhoff construction for quantum propagators with application to diffraction in space and time, Phys. Rev. A 85, 013626 (2012).

[20] A. Goussev, Diffraction in time: An exactly solvable model, Phys. Rev. A 87, 053621 (2013).

[21] A. Goussev, Manipulating quantum wave packets via timedependent absorption, Phys. Rev. A 91, 043638 (2015).

[22] A. del Campo and J. G. Muga, Exact propagators for atom-laser interactions, J. Phys. A: Math. Gen. 39, 14079 (2006).

[23] F. Kottler, Zur theorie der beugung an schwarzen schirmen, Ann. Phys. (Leipzig) 70, 405 (1923).

[24] F. Kottler, Diffraction at a black screen. I. Kirchhoff's theory, Prog. Opt. 4, 281 (1965).

[25] J. F. Nye, J. H. Hannay, and W. Liang, Diffraction by a black half-plane: Theory and observation, Proc. R. Soc. London Ser. A 449, 515 (1995).

[26] C. Brukner and A. Zeilinger, Diffraction of matter waves in space and in time, Phys. Rev. A 56, 3804 (1997).

[27] A. D. Baute, I. L. Egusquiza, and J. G. Muga, Sources of quantum waves, J. Phys. A: Math. Gen. 34, 4289 (2001).
[28] A. del Campo, J. G. Muga, and M. Moshinsky, Time modulation of atom sources, J. Phys. B 40, 975 (2007).

[29] E. Torrontegui, J. Muñoz, Y. Ban, and J. G. Muga, Explanation and observability of diffraction in time, Phys. Rev. A 83, 043608 (2011).

[30] M. Beau and T. C. Dorlas, Three-dimensional quantum slit diffraction and diffraction in time, Int. J. Theor. Phys. 54, 1882 (2015).

[31] B. Navarro, I. L. Egusquiza, J. G. Muga, and G. C. Hegerfeldt, Suppression of Rabi oscillations for moving atoms, Phys. Rev. A 67, 063819 (2003).

[32] G. Scheitler and M. Kleber, On the adiabaticity of continuum states: Tunneling through a time-dependent barrier, Z. Phys. D 9, 267 (1988).

[33] C. M. Fabre, P. Cheiney, G. L. Gattobigio, F. Vermersch, S. Faure, R. Mathevet, T. Lahaye, and D. Guéry-Odelin, Realization of a Distributed Bragg Reflector for Propagating Guided Matter Waves, Phys. Rev. Lett. 107, 230401 (2011).

[34] P. Cheiney, C. M. Fabre, F. Vermersch, G. L. Gattobigio, R. Mathevet, T. Lahaye, and D. Guéry-Odelin, Matter-wave scattering on an amplitude-modulated optical lattice, Phys. Rev. A 87, 013623 (2013).

[35] F. Jendrzejewski, K. Müller, J. Richard, A. Date, T. Plisson, P. Bouyer, A. Aspect, and V. Josse, Coherent Backscattering of Ultracold Atoms, Phys. Rev. Lett. 109, 195302 (2012).

[36] E. J. Heller, Frozen Gaussians: A very simple semiclassical approximation, J. Chem. Phys. 75, 2923 (1981).

[37] See,e.g., L. S. Schulman, Techniques and Applications of Path Integration (Wiley, New York, 1981).

[38] See,e.g., J. Zinn-Justin, Path Integrals in Quantum Mechanics (Oxford University Press, Oxford, 2010).

[39] See,e.g., A. Erdélyi, W. Magnus, F. Oberhettinger, and F. G. Tricomi, Tables of Integral Transforms (McGraw-Hill, New York, 1954), Vol. 1.

[40] See, e.g., I. S. Gradshteyn and I. M. Ryzhik, Table of Integrals, Series, and Products, 7th ed., edited by A. Jeffrey and D. Zwillinger (Elsevier/Academic, Amsterdam, 2007).

[41] J. Crank and P. Nicolson, A practical method for numerical evaluation of solutions of partial differential equations of the heat-conduction type, Math. Proc. Cambridge Philos. Soc. 43, 50 (1947).

[42] A. Marchewka and Z. Schuss, Total absorption in finite time in an $i \delta$ potential, arXiv:quant-ph/0107152.

[43] S. Albeverio, F. Gesztesy, R. Høegh-Krohn, and H. Holden, Solvable Models in Quantum Mechanics, 2nd ed. (AMS Chelsea, Providence, 2005).

[44] M. Calçada, J. T. Lunardi, L. A. Manzoni, and W. Monteiro, Distributional approach to point interactions in one-dimensional quantum mechanics, Front. Phys. 2, 23 (2014). 